\title{
Green's Function for a Hierarchical Self-Avoiding Walk in Four Dimensions
}

\author{
David C. Brydges* \\ University of British Columbia \\ Mathematics Department \\ \#121-1984 Mathematics Road \\ Vancouver, B.C. V6T 1Z2 \\ Canada \\ db5d@math.ubc.ca \\ and \\ Department of Mathematics \\ Kerchof Hall \\ P. O. Box 400137 \\ University of Virginia
}

\author{
John Z. Imbrie \\ Department of Mathematics \\ Kerchof Hall \\ P. O. Box 400137 \\ University of Virginia \\ Charlottesville, VA 22904-4137 \\ ji2k@virginia.edu
}

\begin{abstract}
This is the second of two papers on the end-to-end distance of a weakly selfrepelling walk on a four dimensional hierarchical lattice. It completes the proof that the expected value grows as a constant times $\sqrt{T} \log ^{\frac{1}{8}} T\left(1+O\left(\frac{\log \log T}{\log T}\right)\right)$, which is the same law as has been conjectured for self-avoiding walks on the simple cubic lattice $\mathbb{Z}^{4}$.

Apart from completing the program in the first paper, the main result is that the Green's function is almost equal to the Green's function for the Markov process with no self-repulsion, but at a different value of the killing rate $\beta$ which can be accurately calculated when the interaction is small. Furthermore, the Green's function is analytic in $\beta$ in a sector in the complex plane with opening angle greater than $\pi$.
\end{abstract}

*Research supported by NSF Grant DMS-9706166 


\section{Contents}

\begin{tabular}{llr}
\hline & Introduction & 1
\end{tabular}

\begin{tabular}{lll}
\hline 2 & $\tau$ Isomorphism & 2
\end{tabular}

\begin{tabular}{lll}
\hline 3 & Renormalization Transformations & 6
\end{tabular}

\begin{tabular}{|lr}
\hline Coordinates for Interactions & 10
\end{tabular}

5 Second Order Perturbation Theory 11

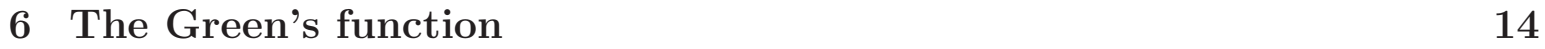

\begin{tabular}{lll}
\hline 7 & Estimates on a Renormalization Group Step & 17
\end{tabular}

\begin{tabular}{|ll|}
\hline A Convolution of Forms and Supersymmetry & 30
\end{tabular}

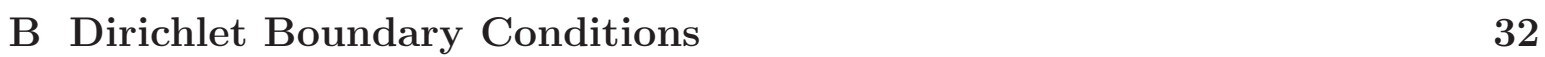

\begin{tabular}{|ll}
\hline C Calculations for Proposition 5.1 & 34
\end{tabular} 


\section{Introduction}

This paper is the second in a series of two papers in which we study the asymptotic end-to-end distance of weakly self-avoiding walk on the four dimensional Hierarchical lattice $\mathcal{G}$. The reader is referred to [BEI92] or the first paper [B]], henceforth referred to as paper I, for definitions of these terms. Results from the first paper have the prefix "I".

In paper I we proved that the self-avoidance causes a $\log ^{1 / 8} T$ correction in the expected end-to-end distance after time $T$ relative to the $\sqrt{T}$ law of a simple random walk. Paper I was devoted to the problem of how to recover the end-to-end distribution by taking the inverse Laplace transform of the Green's function, assuming that the Green's function has certain properties, which are proved in this paper in Theorem 1.1 and Proposition 6.1. These properties are of independent interest and, with minor changes, should also hold for the Green's function for the simple cubic four dimensional lattice. We prove they hold for the hierarchical problem in this paper.

The interacting Green's function is defined by the Laplace transform

$$
G_{\lambda}(\beta, x)=\int_{0}^{\infty} e^{-\beta T} \mathbb{E}_{0}\left(1_{\omega(T)=x} e^{-\lambda \int_{\mathcal{G}} \tau_{x}^{2} d x}\right) d T,
$$

where $\tau_{x}=\tau_{x}^{(T)}$ is the time up to $T$ that $\omega(t)$ is at site $x$. Our main result is Theorem 1.1. It says that the interacting Green's function is almost equal to the Green's function for the Markov process, $\lambda=0$, but at a different value of the $\beta$ parameter which depends on $x$ and $(\beta, \lambda)$ and which can be accurately calculated when $\lambda$ is small. The error in the approximation decays more rapidly than the Green's function because it contains, as a prefactor, the "running coupling constant" $\lambda_{N(x)}$. Furthermore the Green's function is analytic in $\beta$ in a sector in the complex plane with opening angle greater than $\pi$. This very large domain of analyticity seems to be needed for accurately inverting the Laplace transform to calculate the end-to-end distance.

The main theorem refers to domains

$$
\begin{aligned}
& \mathcal{D}_{\beta}=\left\{\beta \neq 0:|\arg \beta|<b_{\beta}\right\} \\
& \mathcal{D}_{\lambda}=\left\{\lambda: 0<|\lambda|<\delta \text { and }|\arg \lambda|<b_{\lambda}\right\} .
\end{aligned}
$$

For details see paper I, but, for example, we can choose $\left(b_{\beta}, b_{\lambda}\right)=\left(\frac{5 \pi}{8}, \frac{\pi}{8}\right)$. The main theorem also refers to a recursion: given $\left(\beta_{0}, \lambda_{0}\right)$ we define the Renormalization Group $(\mathrm{RG})$ recursion $\left(\beta_{j}, \lambda_{j}\right)$ in Sections 3 and 4 and establish the recursive properties in Proposition 6.1. Having established these estimates, we know from paper I that the recursion has various properties. In particular, from Proposition I.1.3, for each $\lambda_{0}$ in the domain there exists a special choice $\beta_{0}=\beta^{c}\left(\lambda_{0}\right)$ for the initial $\beta$ such that the $\mathrm{RG}$ recursion $\left(\beta_{n}^{c}, \lambda_{n}\right)$ is defined for all $n$ and $\beta_{n}^{c} \rightarrow 0$. This should be viewed as a partial description of a stable manifold for the fixed point $(0,0)$, but note that our RG recursion is not autonomous because there are other degrees of freedom, for example, 
the $r$ in Section 4 , which have been projected out in this simplified description. We called $\left(\beta_{n}^{c}, \lambda_{n}\right)$ the critical trajectory. For $\beta_{0}$ some other choice of initial data, we defined

the deviation $\hat{\beta}_{n}:=\beta_{n}-\beta_{n}^{c}$ of its trajectory from the critical trajectory. The main result is

Theorem 1.1. Let $\lambda_{0} \in \mathcal{D}_{\lambda}$ with $\delta$ sufficiently small. Then $G_{\lambda_{0}}\left(\beta_{0}, x\right)$ is analytic in $\beta_{0}$ in the domain $\mathcal{D}_{\beta}+\beta^{c}\left(\lambda_{0}\right)$ and

$$
\left|G_{\lambda_{0}}\left(\beta_{0}, x\right)-G_{0}\left(\beta_{\mathrm{eff}, N(x)}, x\right)\right| \leq O\left(\lambda_{N(x)}\right)\left|G_{0}\left(\beta_{\mathrm{eff}, N(x)}, x\right)\right|
$$

Here $N(x)=\log |x|$ for $x \neq 0, N(0)=0$, and $\beta_{\mathrm{eff}, j}=L^{-2 j} \hat{\beta}_{j}$.

This theorem and Proposition 6.1 are the two results needed to complete the results in paper I.

The paper begins in Section 2 with a review of an isomorphism that recasts the Green's function as an almost Gaussian integral with very special properties (supersymmetry). The virtue of this representation is that it leads to a precise definition of a Renormalization Group ( $\mathrm{RG}$ ) transformation which relates the Green's function with given interaction to a rescaled Green's function with smaller interaction. The RG transformation is defined in Section 3 and its effect on the interaction is further described in Section 4 . To use this RG transformation we need an approximate calculation of its effect on the interaction. This is carried out in Section 5. The proof of the main Theorem 1.1 is in Section 6. All the analysis in this paper is in Section 7. The methods introduced there have several noteworthy features: (i) the demonstration that analysis with supersymmetric integrals containing differential forms $\equiv$ Fermions is possible ; (ii) the use of rotation of contours of integration in the supersymmetric integral to obtain the large domain of analyticity needed for inverting the Laplace transform; and (iii) simultaneous control over behavior in $x$ and behavior in $\beta$ both large and small.

With Steven Evans we wrote an earlier paper [BEI92] on this same model which studied the Green's function but only at the critical value of the killing rate. Here we have opted for some repetition of ideas in that paper because we have since learned that the Grassmann algebras used in [BEI92 are natural differential forms and we wanted to incorporate this insight systematically.

\section{$2 \quad \tau$ Isomorphism}

In a precise sense that we will now review, the differential form

$$
\phi_{x} \bar{\phi}_{x}+\frac{1}{2 \pi i} d \phi_{x} d \bar{\phi}_{x}
$$

represents the time $\tau_{x}$ a finite state Markov process occupies state $x$. The following is a distillation of ideas in papers [McK80, PS80, Lut83, LJ87]. 
The forms $d \phi_{x}, d \bar{\phi}_{x}$ are multiplied by the wedge product. To connect with notation in BEI92, BMM91, we set

$$
\psi_{x}=(2 \pi i)^{-1 / 2} d \phi_{x}, \bar{\psi}_{x}=(2 \pi i)^{-1 / 2} d \bar{\phi}_{x}
$$

where $(2 \pi i)^{-\frac{1}{2}}$ is a fixed choice of square root. Let $\Lambda$ be a finite set. Given any matrix $A_{x y}$ with indices $x, y \in \Lambda$ we define the even differential form

$$
S_{A}=\sum \phi_{x} A_{x y} \bar{\phi}_{y}+\sum \psi_{x} A_{x y} \bar{\psi}_{y}
$$

and then the exponential of this form is defined by the Taylor series

$$
e^{-S_{A}}=e^{-\sum \phi_{x} A_{x y} \bar{\phi}_{y}} \sum \frac{1}{n !}\left(-\sum \psi_{x} A_{x y} \bar{\psi}_{y}\right)^{n} .
$$

The series terminates after finitely many terms because the anticommutative wedge product vanishes if the degree of the form exceeds the real dimension $2|\Lambda|$ of $\mathbb{C}^{\Lambda}$.

By definition $\int_{\mathbb{C}^{\Lambda}}$ vanishes on forms that are of degree less than the real dimension of $\mathbb{C}^{\Lambda}$. For example, taking $|\Lambda|=1$ and $A_{x y}=A>0$, we find that $\int_{\mathbb{C}} e^{-S_{A}}=1$ because only the $n=1$ term in the expansion of the exponential contributes and this term is

$$
-(2 \pi i)^{-1} A \int_{\mathbb{C}} e^{-\phi A \bar{\phi}} d \phi d \bar{\phi}=\frac{A}{\pi} \iint e^{-A\left(u^{2}+v^{2}\right)} d u d v=1,
$$

using $\phi=u+i v$ and $d \phi d \bar{\phi}=-2 i d u d v$. Thus these integrals are self-normalizing. This feature generalizes:

Lemma 2.1. Suppose that $A$ has positive real part, meaning $\operatorname{Re} \sum \phi_{x} A_{x y} \bar{\phi}_{y}>0$ for $\phi \neq 0$. Then

$$
\begin{aligned}
& \int_{\mathbb{C}^{\Lambda}} e^{-S_{A}}=1, \\
& \int_{\mathbb{C}^{\Lambda}} e^{-S_{A}} \phi_{a} \bar{\phi}_{b}=C_{a b},
\end{aligned}
$$

where $C=A^{-1}$.

The second part of the lemma follows from the first part together with the standard fact that the covariance of a normalized Gaussian measure is the inverse of the matrix in the exponent. The first part is a corollary of Lemma 2.2 given below.

Let

$$
\tau_{x}=\phi_{x} \bar{\phi}_{x}+\psi_{x} \bar{\psi}_{x}
$$

and let $\tau$ be the collection $\left(\tau_{x}\right)_{x \in \Lambda}$. Given any smooth function $F(t)$ defined on $\mathbb{R}^{\Lambda}$, we use the terminating Taylor series

$$
F(\tau)=\sum_{\alpha} \frac{1}{\alpha !} F^{(\alpha)}(\phi \bar{\phi})(\psi \bar{\psi})^{\alpha}
$$


to define the form $F(\tau)$, where $\phi \bar{\phi}=\left(\phi_{x} \bar{\phi}_{x}\right)_{x \in \Lambda},(\psi \bar{\psi})^{\alpha}=\prod\left(\psi_{x} \bar{\psi}_{x}\right)^{\alpha_{x}}$. The even degree of $\tau_{x}$ relieves us of any necessity to specify an order for the product over forms.

Supersymmetry: There is a flow on $\mathbb{C}^{\Lambda}$ given by $\phi_{x} \longmapsto \exp (-2 \pi i t) \phi_{x}$. This flow is generated by the vector field $X$ such that $X\left(\phi_{x}\right)=-2 \pi i \phi_{x}$ and $X\left(\bar{\phi}_{x}\right)=2 \pi i \bar{\phi}_{x}$. A form $\omega$ is invariant (under this flow) if it is unchanged by the substitution $\phi_{x} \longmapsto$ $\exp (-2 \pi i t) \phi_{x}$. The Lie derivative $\mathcal{L}_{X} \omega$ of a form $\omega$ is obtained by differentiating with respect to the flow at $t=0$ so invariance is equivalent to $\mathcal{L}_{X} \omega=0$.

Let $i_{X}$ be the interior product with the vector field $X$. The supersymmetry operator Wit92, AB84

$$
Q=d+i_{X}
$$

is an anti-derivation on forms with the property that $Q^{2}=d i_{X}+i_{X} d=\mathcal{L}_{X}$ is the Lie derivative. Therefore $Q^{2}=0$ on forms $\omega$ which are invariant. A form $\omega$ that satisfies the stronger property $Q \omega=0$ is said to be supersymmetric. For example $S_{A}$ is supersymmetric and the derivation property implies that $\exp \left(-S_{A}\right)$ is also supersymmetric. Since $Q \phi_{x} d \bar{\phi}_{x}=d \phi_{x} d \bar{\phi}_{x}+(2 \pi i) \phi_{x} \bar{\phi}_{x}$, there is a form $u_{x}$ such that $\tau_{x}=Q u_{x}$. $S_{A}$ is also in the image of $Q$.

For any form $u$ whose coefficients decay sufficiently rapidly at infinity,

$$
\int_{\mathbb{C}^{\Lambda}} Q u=0
$$

because the integral of $d u$ is zero by Stokes theorem, while the integral of $i_{X} u$ is zero because it cannot contain a form of degree $2|\Lambda|$.

Let $F \in \mathcal{C}_{0}^{\infty}\left(\mathbb{R}^{\Lambda}\right)$. Then $\int e^{-S_{A}} F(\lambda \tau)$ is independent of $\lambda$, because the $\lambda$ derivative has the form

$$
\int_{\mathbb{C}^{\Lambda}} e^{-S_{A}} \sum_{x} F_{t_{x}}(\lambda \tau) Q u_{x}=\int_{\mathbb{C}^{\Lambda}} Q\left(e^{-S_{A}} \sum_{x} F_{t_{x}}(\lambda \tau) u_{x}\right)=0 .
$$

The compact support condition is a simple way to be sure that there are no boundary terms at infinity. Adequate decay of the integrand and its partial derivatives is all that is needed. If the exponential has better decay then there is no need for such a strong condition on $F$. Thus

Lemma 2.2. If $A$ has positive real part and $F$ is smooth on $\mathbb{R}^{\Lambda}$ with bounded derivatives, then

$$
\int_{\mathbb{C}^{\Lambda}} e^{-S_{A}} F(\tau)=F(0)
$$

Part (1) of Lemma 2.1 is obtained when $F=1$.

The following Proposition will be called the $\tau$ isomorphism. It is the main result of this section. 
Proposition 2.3. PS79, McK80 Suppose that A generates a Markov process with killing on first exit from $\Lambda$. Let $\mathbb{E}_{a}(\cdot)$ denote the associated expectation over paths $\omega(t)$ such that $\omega(0)=a$. Let $F \in \mathcal{C}_{0}^{\infty}\left(\mathbb{R}^{\Lambda}\right)$, then

$$
\int_{\mathbb{C}^{\Lambda}} e^{-S_{A}} F(\tau) \phi_{a} \bar{\phi}_{b}=\int_{0}^{\infty} d T \mathbb{E}_{a}\left(F(\tau) 1_{\omega(T)=b}\right) .
$$

On the right-hand side $\tau_{x}=\tau_{x}^{T}$ is the time up to $T$ that the stochastic process $\omega(t)$ is at site $x$,

$$
\tau_{x}^{T}=\int_{0}^{T} 1_{\omega(t)=x} d t
$$

On the left-hand side it is the form $\phi_{x} \bar{\phi}_{x}+\psi_{x} \bar{\psi}_{x}$.

As noted above, the compact support condition on $F$ is stronger than necessary. The left-hand side is a linear combination of integrals that involve finitely many derivatives of $F$. It is still valid if these derivatives have adequate decay at infinity, as will be the case for functions used in this paper.

Proof. We can assume, with no loss of generality, that $\operatorname{Re} \sum \phi_{x} A_{x y} \bar{\phi}_{y}>0$ when $\phi \neq 0$, because both sides are unchanged by

$$
F(t) \mapsto F(t) e^{\kappa \sum t_{x}}, \quad A \mapsto A+\kappa I .
$$

Consider the special case where $F(\tau)=\exp \left(i \sum k_{x} \tau_{x}\right)$. Then

$$
\int_{\mathbb{C}^{\Lambda}} e^{-S_{A}} F(\tau) \phi_{a} \bar{\phi}_{b}=\int_{\mathbb{C}^{\Lambda}} e^{-S_{A-i K}} \phi_{a} \bar{\phi}_{b},
$$

where $K$ is the diagonal matrix $k_{x} \delta_{x y}$. By Lemma 2.1 the right-hand side equals $(A-i K)_{a b}^{-1}$ which is

$$
\int_{0}^{\infty}\left(e^{-T[A-i K]}\right)_{a b}=\int_{0}^{\infty} d T \mathbb{E}_{a}\left(F(\tau) 1_{\omega(T)=b}\right) .
$$

by the Feynman-Kac formula円 and $F(\tau)=\exp \left(i \sum k_{x} \tau_{x}\right)=\exp \left(i \int_{0}^{T} k_{\omega(s)} d s\right)$.

By (2.4) the proposition is proven for $F(\tau)=\exp \left(i \sum k_{x} \tau_{x}\right)$. Both sides of the proposition are linear in $F$ so we can generalize to $F \in \mathcal{C}_{0}^{\infty}$ by substituting the Fourier inversion formula

$$
F(\tau)=(2 \pi)^{-n} \int \hat{F}(k) e^{i \sum k_{x} \tau_{x}} d^{|\Lambda|} k
$$

into $\int \exp \left(-S_{A}\right) F(\tau) \phi_{a} \bar{\phi}_{b}$. Since the $k$ and $\phi$ integrals, by (2.3), are absolutely convergent, the integral over $k$ may be interchanged with the $\phi$ integrals.

\footnotetext{
${ }^{1}$ The Feynman-Kac formula is given in Sim79 for Brownian motion. The proof that uses the Trotter product formula is valid for finite state Markov processes.
} 


\section{Renormalization Transformations}

Since the $\tau$ isomorphism is only applicable when the state space is finite we have to study the Green's function as a limit of processes with finite state spaces.

Let $N$ be a positive integer and let $\mathbb{E}_{0}^{\Lambda}(\cdot)$ be the expectation for the hierarchical Levy process $\omega(t)$ killed on first exit from $\Lambda=\mathcal{G}_{N}$. Define the finite volume interacting Green's function

$$
G_{\lambda}^{\Lambda}(\beta, x)=\int_{0}^{\infty} e^{-\beta T} \mathbb{E}_{0}^{\Lambda}\left(1_{\omega(T)=x} e^{-\lambda \int_{\mathcal{G}} \tau_{x}^{2} d x}\right) d T
$$

When $\lambda=0, G_{\lambda=0}^{\Lambda}(\beta, x)$ is the $\beta$ potential for the hierarchical Levy process $\omega(t)$ killed on first exit from $\Lambda=\mathcal{G}_{N}$. In this section we single out this important object with the notation $U^{\Lambda}(\beta, x)=G_{\lambda=0}^{\Lambda}(\beta, x)$.

Given a bounded smooth function $g(t)$ we define a generalization of the Green's function

$$
G_{g}^{\Lambda}(\beta, x)=\int_{0}^{\infty} e^{-\beta T} \mathbb{E}_{0}^{\Lambda}\left(g^{\Lambda} 1_{\omega(T)=x}\right) d T
$$

where $g^{\Lambda}=\prod_{x \in \Lambda} g\left(\tau_{x}\right)$. When $g$ is the function $g(t)=\exp \left(-\lambda t^{2}\right)$ this is the Green's function (3.1). By the $\tau$ isomorphism,

$$
G_{g}^{\Lambda}(\beta, x)=\int_{\mathbb{C}^{\Lambda}} \mu_{\Lambda}\left(e^{-\beta \tau} g\right)^{\Lambda} \phi_{0} \bar{\phi}_{x}
$$

where $\mu_{\Lambda}$ is the Gaussian form $\exp \left(-S_{A}\right)$ with $A$ equal to the inverse of $U^{\Lambda}$ with $\beta=0$.

Scaling: This is a transformation $x \mapsto L^{-1} x$ that maps the hierarchical lattice to itself by identifying all points that lie in the same ball of diameter $L$ in the hierarchical lattice so that they become a single point in a new hierarchical lattice. Thus it is the canonical projection (of groups), $\mathcal{G}_{N} \rightarrow \mathcal{G}_{N} / \mathcal{G}_{1}$, rewritten as

$$
L^{-1} x=\left(\ldots, x_{3}, x_{2}, x_{1}\right), \quad \text { for } x=\left(\ldots, x_{3}, x_{2}, x_{1}, x_{0}\right),
$$

which maps $\Lambda=\mathcal{G}_{N}$ to $\Lambda / L:=\mathcal{G}_{N-1}$. Associated to these lattices we have manifolds $\mathbb{C}^{\Lambda}$ and $\mathbb{C}^{\Lambda / L}$. A point in $\mathbb{C}^{\Lambda}$ is specified by $\left(\phi_{x}\right)_{x \in \Lambda}$. Scaling therefore maps a point $\phi$ in $\mathbb{C}^{\Lambda / L}$ backward to a point $\mathcal{S} \phi$ in $\mathbb{C}^{\Lambda}$ according to

$$
\mathcal{S} \phi_{x} \equiv(\mathcal{S} \phi)_{x}=L^{-1} \phi_{L^{-1} x}
$$

so $\mathcal{S} \phi$ is constant on cosets $x+\mathcal{G}_{1}$. The prefactor $L^{-1}$ is put there to make the RG map, to be defined below, autonomous. Functions and forms on $\mathbb{C}^{\Lambda}$ are mapped forward. For example, for $x \in \Lambda, d \phi_{x}$ is a form on $\mathbb{C}^{\Lambda}$. Under scaling we get

$$
\mathcal{S}\left(d \phi_{x}\right)=L^{-1} d \phi_{x / L}
$$


which is a form on $\mathbb{C}^{\Lambda / L}$. We define scaling on covariances by

$$
\mathcal{S} C^{\Lambda / L}(x, y) \equiv\left(\mathcal{S} C^{\Lambda / L}\right)(x, y)=L^{-2} C\left(L^{-1} x, L^{-1} y\right)
$$

Covariances are functions on the lattices so they are mapped backwards. To summarize: the direction of maps may appear reversed, but observe that the projection $\Lambda \rightarrow \Lambda / L$ induces a map backwards of manifolds $\mathbb{C}^{\Lambda / L} \rightarrow \mathbb{C}^{\Lambda}$ because $\mathbb{C}^{X}=\operatorname{map}(X \rightarrow \mathbb{C})$ and so forms/functions on the manifold $\mathbb{C}^{\Lambda}$ are mapped forward to forms/functions on the manifold $\mathbb{C}^{\Lambda / L}$.

The renormalization group rests on the following scaling decomposition

$$
U^{\Lambda}(\beta, x)=\mathcal{S} U^{\Lambda / L}\left(L^{2} \beta, x\right)+\Gamma(\beta, x) .
$$

The important properties of $\Gamma$ defined by this formula are that $\Gamma$ is positive semi-definite and finite range. In Appendix $\mathrm{B}$ we prove that

$$
\Gamma(\beta, x)=\frac{1}{1+\beta}\left(1_{\mathcal{G}_{0}}(x)-L^{-4} 1_{\mathcal{G}_{1}}(x)\right) .
$$

$\Gamma$ also has the inessential properties $\sum_{y} \Gamma(\beta, y)=1$ and $\Gamma(\beta, y)=\Gamma\left(\beta, y^{\prime}\right)$ for $y, y^{\prime} \neq 0$ which lead to simplifications specific to this model, notably Lemma 3.5 .

Let $C_{x y}$ with $x, y \in \Lambda$ be an invertible matrix and let $A$ be the inverse of $C_{x y}$. Define $\mu_{C}=\exp \left(-S_{A}\right)$. According to Lemma 2.1, $\int \mu_{C} \phi_{a} \bar{\phi}_{b}=C_{a b}$ whenever the inverse $A$ has positive-definite real part. The $\Gamma$ appearing in (3.4) is only positive semi-definite because, as an operator on $\mathbb{C}^{\Lambda}$, it has the kernel $\Xi^{\perp}$ consisting of all $\phi_{x}$ that are constant on cosets $x+\mathcal{G}_{1}$, because $\sum_{y} \Gamma_{x-y}=0$. Let $\Xi \subset \mathbb{C}^{\Lambda}$ be the subspace orthogonal to the kernel of $\Gamma$. $\Gamma$ restricts to the invariant subspace $\Xi$. The restricted $\Gamma$ is positive-definite and invertible. We choose coordinates $\zeta$ in $\Xi$ by picking any basis and define $\mu_{\Gamma}$ as a form on $\Xi$ using the inverse of $\Gamma$ computed in this basis. The form $\mu_{\Gamma}$ is independent of this choice of basis because forms are coordinate invariant. The matrix $\mathcal{S} U^{\Lambda / L}$ also has no inverse, but by the same reasoning defines a form $\mu_{\mathcal{S} U^{\Lambda / L}}$ on $\Xi^{\perp}$. The action of $\mathcal{S}$ on covariances was defined so that

$$
\int \mu_{\mathcal{S} U} u=\int \mu_{U} \mathcal{S} u
$$

where $U=U^{\Lambda / L}$ and $u$ is a form on on $\Xi^{\perp} \approx \mathbb{C}^{\Lambda / L}$.

Let $g(\phi)=\sum g_{\alpha, \bar{\alpha}}(\phi) d \phi^{\alpha} d \bar{\phi}^{\bar{\alpha}}$ be a form on $\mathbb{C}^{\Lambda}$. Then $g(\phi+\zeta)$ is a form on $\Xi^{\perp} \times \Xi$ defined by pullback, i.e., substituting $\phi+\zeta$ for $\phi$. Define the form $\mu_{\Gamma} * g$ on $\Xi^{\perp}$ by

$$
\mu_{\Gamma} * g(\phi)=\int_{\Xi} \mu_{\Gamma}(\zeta) g(\phi+\zeta) .
$$

The scale decomposition (3.4) leads to the convolution property

$$
\int_{\mathbb{C}^{\Lambda}} \mu_{U}(\phi) F(\phi)=\int_{\Xi^{\perp}} \mu_{\mathcal{S} U}(\phi) \mu_{\Gamma} * F(\phi)
$$

which is valid for $F(\phi)$ any smooth bounded form. This claim follows by changing variables $(u, v)=(\phi+\zeta, \zeta)$ and integrating out $v$ using Corollary A.3. 
Definition 3.1. Define the linear operator $\mathbb{T}_{\beta}$ that maps forms on $\mathbb{C}^{\Lambda}$ to forms on $\mathbb{C}^{\Lambda / L}$ by $\mathbb{T}_{\beta} u=\mathcal{S} \mu_{\Gamma(\beta)} * u$. $\mathbb{T}_{\beta}$ is called a renormalization group $(R G)$ transformation.

\section{Proposition 3.2.}

$$
\int_{\mathbb{C}^{\Lambda}} \mu_{\Lambda} e^{-\beta \int \tau} u=\int_{\mathbb{C}^{\Lambda / L}} \mu_{\Lambda / L} e^{-\left(L^{2} \beta\right) \int \tau} \mathbb{T}_{\beta} u
$$

Proof. By the $\tau$ isomorphism, the covariance of the Gaussian $\mu_{\Lambda} \exp \left(-\beta \int \tau\right)$ is the same as the covariance of $\mu_{U}$ when $U$ has parameters $\Lambda, \beta$. Therefore the two Gaussian forms are equal. By the convolution property (3.7), $\int \mu_{U} u=\int \mu_{\mathcal{S} U} \mu_{\Gamma} * u$ where $U$ in $\mathcal{S} U$ has scaled parameters $\Lambda / L$ and $L^{2} \beta$. Apply (3.6).

Lemma 3.3. $\mathbb{T}_{\beta}$ commutes with the supersymmetry operator $Q$ defined in (2.9). Furthermore, if $u$ is an even supersymmetric form on $\mathbb{C}^{x+\mathcal{G}_{1}}$, then there is a unique function f such that $\mathbb{T}_{\beta} u=f\left(\tau_{x}\right)$.

Proof. $Q$ commutes with $\mathcal{S}$ because $\mathcal{S}$ is a pullback. By Lemma A.2, $Q$ also commutes with integrating out. Thus $\left[\mathbb{T}_{\beta}, Q\right]=0$ because $\mu_{\Gamma}$ is supersymmetric. The existence of $f$ follows from Lemma A.4.

Let $X$ be a subset of $\Lambda$. A form $F_{X}$ is said to be localized in $X$ if it is a form on $\mathbb{C}^{X}$. Since Gaussian random variables are independent if their covariance vanishes, we have the independence property

$$
\mu_{\Gamma} *\left(F_{X} G_{Y}\right)=\left(\mu_{\Gamma} * F_{X}\right)\left(\mu_{\Gamma} * G_{Y}\right)
$$

whenever the hierarchical distance between $X$ and $Y$ exceeds the range of $\Gamma$. Given forms $g_{x}$ localized at single sites $\{x\}$ let

$$
g^{X}=\prod_{x \in X} g_{x}
$$

Then the independence property implies

$$
\mathbb{T}_{\beta} g^{\Lambda}=\prod_{x \in \Lambda / L} \mathbb{T}_{\beta} g^{x+\mathcal{G}_{1}}
$$

$\mathbb{T}_{\beta} g^{x+\mathcal{G}_{1}}$ is a form on $\mathbb{C}^{\{x\}}$. By Lemma 3.3, it has the form $g_{\text {new }}\left(\tau_{x}\right)$ for some function $g_{\text {new }}(t)$ on $\mathbb{R}$. This is a marvelous property of the hierarchical lattice because it means that the RG map preserves the multiplicativity of the interaction:

$$
\mathbb{T}_{\beta} g^{\Lambda}=g_{\text {new }}^{\Lambda / L},
$$

and therefore $\mathbb{T}_{\beta}$ can be described by the map $g \rightarrow g_{\text {new }}$. This has come about because the hierarchical topology has no overlapping neighborhoods: any pair of neighborhoods are either disjoint or nested. 
However, there is some redundancy in the pair $(\beta, g)$ : as noted in (2.3), the Green's function $G_{g}(\beta, x)$ depends only on the combination $g(t) \exp (-\beta t)$. We will remove this redundancy by imposing the normalization condition

$$
g(t)=1+O\left(t^{2}\right) \text { as } t \rightarrow 0 .
$$

This normalization assumes that $g_{\text {new }}(0)=1$. This is true for the initial interaction $g(t)=\exp \left(-\lambda t^{2}\right)$ and, by Lemma 2.2, it also holds for $g_{\text {new }}$. After each map by $\mathbb{T}_{\beta}$, which, referring to Proposition 3.2 , takes $(\beta, g)$ to $\left(L^{2} \beta, g_{\text {new }}\right)$, the pair $\left(L^{2} \beta, g_{\text {new }}\right)$ is replaced by an equivalent pair $\left(\beta^{\prime}, g^{\prime}\right)=\left(L^{2} \beta+\nu^{\prime}, \exp \left(\nu^{\prime} t\right) g_{\text {new }}\right)$ to restore (3.9).

Definition 3.4. Let $g$ be a smooth bounded function on $\mathbb{R}$ and let $g_{x}=g\left(\tau_{x}\right)$. $T_{\beta} g$ is the function on $\mathbb{R}$, given by

$$
T_{\beta} g\left(\tau_{x}\right)=e^{\nu^{\prime} t} \mathbb{T}_{\beta} g^{x+\mathcal{G}_{1}},
$$

where $\nu^{\prime}$ is chosen so that $T_{\beta} g(t)=1+O\left(t^{2}\right)$.

In (3.3) there is also the factor $\phi_{0} \bar{\phi}_{x}$. By the independence property (3.8), the RG acts on each of the factors $\phi_{0}$ and $\phi_{x}$ independently if $|x|>L$. In fact, in this model, the RG acts simply by scaling:

Lemma 3.5. Suppose that $g=a(\phi)+b(\phi) d \phi d \bar{\phi}$ is an even form on $\mathbb{C}$. Define $g_{x}$ by replacing $\phi$ by $\phi_{x}$, then

$$
\mathbb{T}_{\beta} g^{x+\mathcal{G}_{1}} \phi_{x}=\left(\mathbb{T}_{\beta} g^{x+\mathcal{G}_{1}}\right) \mathcal{S} \phi_{x}
$$

Proof. Let $G=g^{x+\mathcal{G}_{1}}$ with $L^{-2} \phi_{x / L}+\zeta_{x}$ substituted in place of $\phi_{x}$, then

$$
\mathbb{T}_{\beta} g^{x+\mathcal{G}_{1}} \phi_{x}=\left(\mathcal{S} \int \mu_{\Gamma} G\right) \mathcal{S} \phi_{x}+\mathcal{S} \int \mu_{\Gamma} G \zeta_{x}
$$

so we have to prove that $\int \mu_{\Gamma} G \zeta_{x}=0$. By $\mathcal{G}_{1}$ invariance, $\int \mu_{\Gamma} G \zeta_{x}=\int \mu_{\Gamma} G \zeta_{y}$ for all $y \in x+\mathcal{G}_{1}$. Since $\zeta \in \Xi, \sum_{y \in \mathcal{G}} \zeta_{y}=0$.

By (3.3), Proposition 3.2 and Lemma 3.5 we have proved that

Proposition 3.6. For $|x|>L$,

$$
G_{g}^{\Lambda}(\beta, x)=\mathcal{S} G_{g^{\prime}}^{\Lambda / L}\left(\beta^{\prime}, x\right),
$$

where $g^{\prime}=T_{\beta} g$ and $\beta^{\prime}=L^{2} \beta+\nu^{\prime}$ with $\nu^{\prime}$ as in Definition 3.4.

What happens if $|x| \leq L$ ? The transformation of the factor $\phi_{0} \bar{\phi}_{x}$ is no longer simple, but the next result says that it reproduces itself together with supersymmetric corrections.

Lemma 3.7. If $u$ is a smooth supersymmetric even form on $\mathbb{C}^{\mathcal{G}_{1}}$, then there are unique functions $f_{1}, f_{2}$ such that $\mathbb{T}_{\beta} u \phi_{0} \bar{\phi}_{x}=f_{1}\left(\tau_{0}\right)+f_{2}\left(\tau_{0}\right) \phi_{0} \bar{\phi}_{0}$. 
Proof. Let $v=\mathbb{T}_{\beta} u \phi_{0} \bar{\phi}_{x}$ and $w=Q v$, then $w$ is a supersymmetric form of odd degree. By Lemma A.4, $w=a(\tau)(\phi d \bar{\phi}+\bar{\phi} d \phi)$. The solutions of $w=Q v$ are $v=-(2 \pi i)^{-1} a(\tau) \phi \bar{\phi}+b(\tau)$. $a$ and $b$ are unique because the degree zero part of $v$ determines the combination $-(2 \pi i)^{-1} a(t) t+b(t)$ and the degree two part determines $-(2 \pi i)^{-1} a^{\prime}(t) t+b^{\prime}(t)$.

\section{Coordinates for Interactions}

The parameters in the Green's function (3.2) are $\beta$, a smooth function $g$, and the volume $\Lambda . g$ defines a coupling constant $\lambda$ and a smooth function $r(t)$ by

$$
g(t)=e^{-\lambda t^{2}}+r(t) \text { with } r(t)=O\left(t^{3}\right) \text { as } t \rightarrow 0,
$$

because by Definition 3.4, $\beta$ is adjusted so that $g(t)=1+O\left(t^{2}\right) . r$ will be called the remainder.

Consequently, we may describe the map $g \rightarrow T_{\beta} g$ by its action on the parameters $\beta, \lambda, r \rightarrow \beta^{\prime}, \lambda^{\prime}, r^{\prime}$, where $\beta^{\prime}, \lambda^{\prime}$ and $r^{\prime}$ solve

$$
e^{-L^{2} \beta \tau} \mathbb{T}_{\beta}\left(e^{-\lambda \tau^{2}}+r\right)^{\mathcal{G}_{1}}=e^{-\beta^{\prime} \tau}\left(e^{-\lambda^{\prime} \tau^{2}}+r^{\prime}\right), \quad r^{\prime}(t)=O\left(t^{3}\right) .
$$

Iteration of this map defines a finite sequence $\left(\beta_{j}, \lambda_{j}, r_{j}, \Lambda_{j}\right)_{j=0, \ldots, N-1}$. The sequence terminates because the initial $\Lambda_{0}=\mathcal{G}_{N}$ is scaled down by $L$ with each RG map and eventually becomes $\mathcal{G}_{1}$. Then there is one final integration. This sequence exists for any initial choice of parameters with $\operatorname{Re} \lambda_{0}>0$ because $\Lambda_{0}$ is finite.

This hierarchical model has the nice feature that enlarging the initial volume $\Lambda_{0}$ merely extends the sequence - the longer sequence coincides with the shorter for shared indices $j$. Therefore the sequences consistently extend to an infinite sequence.

In (3.3) we rewrite the observables $\phi_{0}$ and $\phi_{x}$ as if they were part of the interaction by

$$
g^{\Lambda} \phi_{0} \bar{\phi}_{x}=\frac{d}{d \gamma} g_{\left.\right|_{0}}^{\Lambda} e^{\gamma \mathcal{O}},
$$

with $\mathcal{O}=b_{1} \phi_{0} \bar{\phi}_{x}$ and $b_{1}=1$. Then Lemma 3.5 asserts that $\mathbb{T}_{\beta}$ acts on $\gamma \mathcal{O}$ at order $\gamma$ by $x \rightarrow L^{-1} x$ and $b_{1} \rightarrow L^{-2} b_{1}$ to produce iterates $x_{j}:=L^{-j} x$ and $b_{i, j}:=L^{-2 j} b_{1}$ for $j=1, \ldots, N(x)-1$, such that $\left|x_{j}\right| \geq L . N(x)$ is the number of iterations, $\log _{L}|x|$, that are needed to scale $x$ to 0 . For $j \geq N(x)-1$, Lemma 3.7 asserts that $\mathrm{T}_{j}:=\mathrm{T}_{\beta_{j}}$ maps $\gamma \mathcal{O}_{j}$ into

$$
\mathcal{O}_{j+1}=f_{1, j+1}\left(\tau_{0}\right)+f_{2, j+1}\left(\tau_{0}\right) \phi_{0} \bar{\phi}_{0} .
$$

In this and subsequent calculations functions of $\gamma$ are identified with their linearizations because we only need to know the derivative with respect to $\gamma$ at $\gamma=0$. The Green's 
function can be accurately calculated without complete knowledge of $f_{1, j}$ and $f_{2, j}$. To this end we define

$$
v_{x}= \begin{cases}\lambda \tau_{x}^{2} & \text { if } x \neq 0 \\ \lambda \tau_{0}^{2}-\gamma\left(b_{0}+b_{1} \phi_{0} \bar{\phi}_{0}+b_{2} \tau_{0} \phi_{0} \bar{\phi}_{0}+b_{3} \tau_{0}\right) & \text { if } x=0\end{cases}
$$

and consider the action of $\mathbb{T}_{\beta}$ on $g^{\Lambda}$ when $g_{x}=e^{-v_{x}}+r_{x}$. Part of the observable $\mathcal{O}$ is in $v$, and the rest of it, which is the part we will not need to calculate in detail, is in $r$. The split is uniquely determined by

Definition 4.1. Let $r$ be a form localized at a single lattice site. We say $r$ is normalized if $\frac{d^{q}}{d t^{q}} r_{t}=0$ at $t=0$ for $q=0,1, \ldots, 5$, where $r_{t}$ is defined by replacing $\phi$ by $t \phi$ in $r$ including in $d \phi$ and $d \bar{\phi}$.

The interaction $v$ is said to be normalized if $v=\lambda \tau^{2}+\gamma \mathcal{O}$ for some $\lambda$ and $\mathcal{O}$ is an even polynomial form of degree less than or equal to four.

The action of $\mathbb{T}_{\beta}$ on $g^{\Lambda}$ is now completely described by the action on parameters

$$
(\beta, \lambda, b, r, \Lambda) \rightarrow\left(\beta^{\prime}, \lambda^{\prime}, b^{\prime}, r^{\prime}, \Lambda^{\prime}\right)
$$

where $b=\left(b_{0}, b_{1}, b_{2}, b_{3}\right)$. The Green's function is

$$
\begin{gathered}
G_{\lambda_{0}}^{\Lambda_{0}}\left(\beta_{0}, x\right)=\frac{d}{d \gamma_{\left.\right|_{0}}} \int \mu_{\Lambda_{0}}\left(e^{-\beta_{0} \tau-\lambda_{0} \tau^{2}}\right)^{\Lambda_{0}} e^{\gamma \phi_{0} \bar{\phi}_{x}} \\
=\frac{d}{d \gamma_{\left.\right|_{0}}} \int \mu_{\mathcal{G}_{1}}\left(e^{-\beta_{N-1} \tau}\left[e^{-v_{N-1}}+r_{N-1}\right]\right)^{\mathcal{G}_{1}} \\
=: b_{0, N},
\end{gathered}
$$

because the final integration with respect to $\mu_{\mathcal{G}_{1}}$ is being considered to be a final $\mathrm{RG}$ mape followed by setting $\phi$ and $d \phi$ to zero so that only the $b_{0}$ part of $v$ ends up in the final result.

Note that the normalization condition is designed so that there is no $\gamma$ dependence in the sequence $\left(\beta_{j}, \lambda_{j}\right)$.

A surprising fact is that the $b_{3} \tau$ term never plays any role beyond being there! No term of the form $\gamma F(\tau)$ with $F(0)=0$ contributes to the Green's function because $(d / d \gamma)_{0} \int \mu G(\tau) \exp (\gamma F(\tau))=G(0) F(0)$ by Lemma 2.2. For this reason, we leave out the $b_{3}$ terms in the rest of this paper.

\section{$5 \quad$ Second Order Perturbation Theory}

We have shown that the $\mathrm{RG}$ induces a map from parameters $(\beta, \lambda, b, r)$ to $\left(\beta^{\prime}, \lambda^{\prime}, b^{\prime}, r^{\prime}\right)$. We will also write $v \rightarrow v^{\prime}$ recalling that $v$ is determined by (4.2). In this section we

\footnotetext{
${ }^{2}$ with $\Gamma$ replaced by $U^{\mathcal{G}_{1}}$
} 
construct an approximation

$$
v \rightarrow \tilde{v}, \quad(\beta, \lambda, b) \rightarrow(\tilde{\beta}, \tilde{\lambda}, \tilde{b})
$$

to the exact map using second order perturbation theory in powers of $\lambda$. This approximation plays a major role in determining the $\log ^{\frac{1}{8}}$ corrections in the end-to-end distance of the interacting walk.

Notation:

$$
B=1-L^{-4}, \quad B_{p}=\int \Gamma^{p}(y) d y
$$

$B_{p}$ is a function of $\beta$ through $\Gamma$. In particular,

$$
B_{1}=0, \quad B_{2}=B(1+\beta)^{-2}, \quad \Gamma(0)=B(1+\beta)^{-1} .
$$

We will show that the second order approximation to $(\beta, \lambda) \rightarrow\left(\beta^{\prime}, \lambda^{\prime}\right)$ is

$$
\begin{aligned}
& \tilde{\beta}=L^{2}\left(\beta+2 \Gamma(0) \lambda+O\left(\Gamma^{3}\right) \lambda^{2}\right) \\
& \tilde{\lambda}=\lambda-8 B_{2} \lambda^{2},
\end{aligned}
$$

where $O\left(\Gamma^{p}\right)$ is an analytic function ${ }^{\beta}$ of $\beta$ and $\lambda$ that is bounded in absolute value by $c|1+\beta|^{-p}$, for $|\lambda|$ bounded.

Likewise, the parameters $b$ have the approximate recursion

$$
\begin{aligned}
& \tilde{b}_{0, j+1}=b_{0, j}+\Gamma_{j}\left(x_{j}\right) b_{1, j}+O\left(\Gamma_{j}^{3}\right) \lambda_{j} b_{1, j}+O\left(\Gamma_{j}^{2}\right) b_{2, j} \\
& \tilde{b}_{1, j+1}=L^{-2}\left[b_{1, j}+O\left(\Gamma_{j}^{2}\right) \lambda_{j} b_{1, j}+O\left(\Gamma_{j}\right) b_{2, j}\right] \\
& \tilde{b}_{2, j+1}=L^{-4}\left[b_{2, j}+O\left(\Gamma_{j}^{2}\right) \lambda_{j} b_{2, j}\right],
\end{aligned}
$$

where the $j$ subscript on $\Gamma$ means that $\beta=\beta_{j}$. All the terms involving $\Gamma$ vanish for $j<N(x)-1$. See Section 4 .

Proposition 5.1. Let $r_{\text {main }}=\mathbb{T}_{\beta}\left(e^{-v}\right)^{\mathcal{G}_{1}}-e^{-\tilde{v}-\tilde{\nu} \tau}$ with $\tilde{\nu}=\tilde{\beta}-L^{2} \beta$. Then $r_{\text {main }}=$ $O\left(\lambda^{3}\right)+O\left(\lambda^{2} \gamma\right)$ as a formal series in powers of $\lambda$.

To prove Proposition 5.1 we introduce the following Laplacian

$$
\Delta_{\Gamma}=\sum_{x, y} \Gamma(x-y)\left(\frac{\partial}{\partial \phi_{x}} \frac{\partial}{\partial \bar{\phi}_{y}}+\frac{\partial}{\partial \psi_{x}} \frac{\partial}{\partial \bar{\psi}_{y}}\right) .
$$

The partial derivatives $\partial / \partial \psi_{x}$ are formal anti-derivatives (equivalently, interior products with vector fields dual to the forms $\psi_{x}$ ). Let $F$ be a smooth bounded form, then

$$
\mu_{t \Gamma} * F=F+t \Delta_{\Gamma} F+O\left(t^{2}\right) \text { as } t \rightarrow 0
$$

\footnotetext{
${ }^{3}$ They are integrals of $p$ or more covariances, $\Gamma(\beta)$ and polynomial in $\lambda$. They can be computed explicitly using Feynman diagrams as in Appendix $\mathrm{C}$.
} 
Given forms $X, Y$ we define a new form $X \stackrel{\leftrightarrow}{\Delta}_{\Gamma} Y$ by

$$
\Delta_{\Gamma} X Y=\left(\Delta_{\Gamma} X\right) Y+X \Delta_{\Gamma} Y+X \overleftrightarrow{\Delta}_{\Gamma} Y
$$

Therefore, denoting partial derivatives with respect to $\phi_{x}$ and $\bar{\phi}_{x}$ by subscripts,

$$
X \stackrel{\leftrightarrow}{\Delta}_{\Gamma} Y=\sum_{x, y} \Gamma(x-y)\left(X_{\phi_{x}} Y_{\bar{\phi}_{y}}+X_{\bar{\phi}_{x}} Y_{\phi_{y}}+(-1)^{\operatorname{sgn}(X)} X_{\psi_{x}} Y_{\bar{\psi}_{y}}-(-1)^{\operatorname{sgn}(X)} X_{\bar{\psi}_{x}} Y_{\psi_{y}}\right)
$$

Thus we can define $X \overleftrightarrow{\Delta}_{\Gamma} \overleftrightarrow{\Delta}_{\Gamma} Y$ by applying the second $\stackrel{\leftrightarrow}{\Delta}_{\Gamma}$ to each term on the righthand side of this equation.

A polynomial form is a form whose coefficients are polynomials in $\phi$ and $\bar{\phi}$. The essential property of such forms is that they are annihilated by $\Delta_{\Gamma}^{j}$ for $j>>1$.

Let $V$ be any polynomial form and set

$$
V_{t}=e^{t \Delta_{\Gamma}} V, \quad \mathcal{L}=\frac{\partial}{\partial t}-\Delta_{\Gamma}
$$

Then $V_{t}$ has the important property $\mathcal{L} V_{t}=0$.

Lemma 5.2. For any polynomial form $V$,

$$
Q_{t}=\frac{1}{2} \sum_{j \geq 1} \frac{1}{j !} V_{t} \stackrel{\leftrightarrow}{\Delta}_{t \Gamma}^{j} V_{t}
$$

satisfies

$$
\mathcal{L}\left[-V_{t}+Q_{t}\right]=\frac{1}{2} V_{t} \stackrel{\leftrightarrow}{\Delta}_{\Gamma} V_{t}
$$

Proof. Since $V$ is a polynomial form, the sum over $j$ terminates after finitely many terms, so $Q$ is defined. Furthermore $\mathcal{L} V_{t}=0$. Therefore,

$$
\begin{gathered}
\mathcal{L}\left(V_{t} \stackrel{\leftrightarrow}{\Delta}_{t \Gamma} V_{t}\right)=V_{t} \stackrel{\leftrightarrow}{\Delta}_{\Gamma} V_{t}-V_{t} \overleftrightarrow{\Delta}_{\Gamma} \stackrel{\leftrightarrow}{\Delta}_{t \Gamma} V_{t} \\
\mathcal{L}\left(\frac{1}{2 !} V_{t} \stackrel{\leftrightarrow}{\Delta}_{t \Gamma}^{2} V_{t}\right)=V_{t} \overleftrightarrow{\Delta}_{\Gamma} \overleftrightarrow{\Delta}_{t \Gamma} V_{t}-\frac{1}{2 !} V_{t} \overleftrightarrow{\Delta}_{\Gamma} \stackrel{\leftrightarrow}{\Delta}_{t \Gamma}^{2} V_{t}
\end{gathered}
$$

together with similar equations for the remaining terms in $Q_{t}$. Add these equations.

Lemma 5.3. Let $\hat{V}_{t}=\left[V_{t}-Q_{t}\right]_{t=1}$. Then $\mu_{\Gamma} * e^{-V}-e^{-\hat{V}_{t}}=O\left(V^{3}\right)$ where $O\left(V^{3}\right)$ means that the formal power series in powers of $\alpha$ obtained by replacing $V$ by $\alpha V$ in the left-hand side is $O\left(\alpha^{3}\right)$.

We call $\hat{V}_{1}$ the second order perturbative effective interaction. 
Proof. We use the Duhamel formula: Let $W_{t}$ be any smooth family of forms with $W_{0}=\exp (-V)$. Then, using (5.6),

$$
\begin{aligned}
\mu_{\Gamma} * e^{-V}-W_{1} & =\int_{0}^{1} d t \frac{d}{d t} \mu_{(1-t) \Gamma} * W_{t} \\
& =\int_{0}^{1} d t \mu_{(1-t) \Gamma} * \mathcal{L} W_{t}
\end{aligned}
$$

Choose $W_{t}=\exp \left(-\hat{V}_{t}\right)$. By Lemma $5.2 \mathcal{L} W_{t}=O\left(\lambda^{3}\right)$ because

$$
\begin{aligned}
\mathcal{L} e^{\hat{V}_{t}} & =e^{\hat{V}_{t}}\left(\frac{1}{2} V_{t} \stackrel{\leftrightarrow}{\Delta}_{\Gamma} V_{t}-\frac{1}{2} \hat{V}_{t} \stackrel{\leftrightarrow}{\Delta}_{\Gamma} \hat{V}_{t}\right) \\
& =e^{\hat{V}_{t}}\left(Q_{t} \stackrel{\leftrightarrow}{\Delta}_{\Gamma} V_{t}-\frac{1}{2} Q_{t} \stackrel{\leftrightarrow}{\Delta}_{\Gamma} Q_{t}\right)=O\left(V^{3}\right)
\end{aligned}
$$

Proof of Proposition 5.1. By Lemma 5.3 applied to $V:=\int_{x+\mathcal{G}_{1}} v_{y} d y$, with $v_{y}$ as defined in (4.2)

$$
e^{-L^{2} \beta \tau_{x}} \mathbb{T}_{\beta} e^{-V}=e^{-L^{2} \beta \tau_{x}} \mathcal{S} e^{-\hat{V}_{1}}=e^{-L^{2} \beta \tau_{x}} e^{-\mathcal{S} \hat{V}_{1}}
$$

$\mathcal{S} \hat{V}_{1}$ is a priori a polynomial of degree six, but in fact the degree six part vanishes because it contains, from $\stackrel{\leftrightarrow}{\Delta}$, a factor $B_{1}=0$. Therefore, $\mathcal{S} \hat{V}_{1}$ is a polynomial form of degree four. It contains a part $\tilde{\nu} \tau_{x}$ which is absorbed into $\tilde{\beta}$, so that $\tilde{\beta}=L^{2} \beta+\tilde{\nu}$ and the remaining part $\mathcal{S} \hat{V}_{1}-\tilde{\nu} \tau_{x}=: \tilde{v}_{x}$ is in the form (4.2) with coefficients $(\tilde{\lambda}, \tilde{b})$.

Details for deriving the formulas (5.3, 5.4) for $(\lambda, b)$ are in Appendix C. The main points are as follows: Suppose coefficients $b, \lambda$ are assigned minus the degree of the monomial they preface. Thus $b_{0}$ has degree zero, $b_{1}, \nu$ have degree -2 and $b_{2}, \lambda$ have degree -4 . Let $\Gamma$ have degree 2 . Then, for example, a term such as $b_{2} \lambda O\left(\Gamma^{2}\right)$ can appear in the right-hand side of the $b_{2}$ recursion because it has degree -2 which equals the degree of $b_{2}$. There are certain terms that do not appear because they contain a factor $\Gamma$ in the form $B_{1}=\sum \Gamma(y)=0$. In fact, for this reason the $b$ recursion is triangular.

The Large Field Problem: We are confining ourselves at present to formal power series statements because $W_{t}=\exp \left(-\hat{V}_{t}\right)$ is not integrable: unlike $\mathcal{S} \hat{V}$, the sixth degree part of $\hat{V}$ does not vanish and $W_{t}$ consequently fails to be integrable. When we prove estimates on remainders in Proposition 7.8, we will use another choice of $W_{t}$ which is the same up to order $O\left(V^{3}\right)$.

\section{The Green's function}

In this section we will prove the main result in this paper, Theorem 1.1. 
Recall that in paper I we introduced the enlarged domains

$$
\begin{aligned}
& \overline{\mathcal{D}}_{\beta}=\left\{\beta \neq 0:|\arg \beta|<b_{\beta}+\frac{1}{4} b_{\lambda}+\epsilon\right\} \\
& \overline{\mathcal{D}}_{\beta}(\rho)=\overline{\mathcal{D}}_{\beta}+\mathcal{B}(\rho) \text { with } \mathcal{B}(\rho)=\{\beta:|\beta|<\rho\} \\
& \overline{\mathcal{D}}_{\lambda}=\left\{\lambda: \quad 0<|\lambda|<\bar{\delta} \text { and }|\arg \lambda|<b_{\lambda}+\epsilon\right\} .
\end{aligned}
$$

We will need

Proposition 6.1. Let $\left(\beta_{0}, \lambda_{0}\right)$ be in the domain $\overline{\mathcal{D}}_{\beta}\left(\frac{1}{2}\right) \times \overline{\mathcal{D}}_{\lambda}$ with $\bar{\delta}$ sufficiently small. The sequence $\left(\beta_{j}, \lambda_{j}\right)_{j=0,1, \ldots, M}$ is such that

$$
\begin{aligned}
& \lambda_{j+1}=\lambda_{j}-\frac{8 B \lambda_{j}^{2}}{\left(1+\beta_{j}\right)^{2}}+\epsilon_{\lambda, j}, \\
& \beta_{j+1}=L^{2}\left[\beta_{j}+\frac{2 B}{1+\beta_{j}} \lambda_{j}\right]+\epsilon_{\beta, j},
\end{aligned}
$$

where the $\epsilon_{\lambda, j}, \epsilon_{\beta, j}$ defined by these equations are analytic functions of $\left(\beta_{0}, \lambda_{0}\right)$ satisfying

$$
\begin{aligned}
& \left|\epsilon_{\lambda, j}\right| \leq c_{L}\left|\lambda_{j}\right|^{3}\left|1+\beta_{j}\right|^{-1}, \\
& \left|\epsilon_{\beta, j}\right| \leq c_{L}\left|\lambda_{j}\right|^{2}\left|1+\beta_{j}\right|^{-2} .
\end{aligned}
$$

Here $B=1-L^{-4}$ and $M$ is the first integer such that $\left(\beta_{M}, \lambda_{M}\right)$ is not in the domain $\overline{\mathcal{D}}_{\beta}\left(\frac{1}{2}\right) \times \overline{\mathcal{D}}_{\lambda}$. If no such integer exists, then $M=\infty$.

The formulas (6.1) were already obtained in (5.3). The new content is the estimate on the errors.

Recall also that there are observable parameters $b_{j}:=\left(b_{0, j}, b_{1, j}, b_{2, j}\right)$. Let $\epsilon_{*, j}=$ $b_{*, j+1}-\tilde{b}_{*, j+1}$ where $*=0,1,2$, be the errors between the exact recursions for these parameters and the second order perturbative recursions, defined in (5.4). Since we defined the observable to be a derivative at $\gamma=0$, we may suppose, without loss of generality, that $\epsilon_{*, j}$ are linear in $b_{j}$. Higher order terms will drop out when $\gamma$ is set to zero. $O\left(\Gamma_{j}^{p}\right)$ denotes an analytic function of $\beta_{0}$ and $\lambda_{0}$ which is defined on $\overline{\mathcal{D}}_{\beta}\left(\frac{1}{2}\right) \times \overline{\mathcal{D}}_{\lambda}$ and bounded in absolute value by $c_{L}\left|1+\beta_{j}\right|^{-p}$.

Proposition 6.2. For $M \geq j \geq N(x)-1$ and $q=0,1,2$,

$$
\left|\epsilon_{q, j}\right| \leq O\left(\Gamma_{j}^{3-q}\right)\left|\lambda_{j}\right|^{2}\left|b_{j}\right|
$$

where $\left|b_{j}\right|:=\left|b_{1, j}\right|+\left|b_{2, j}\right|$. 
These two theorems will be proved in the next section. In the next proof $c_{L}$ denotes constants chosen after $L$ is fixed, whereas $c$ denotes constants chosen before $L$ is fixed. The values of these constants are not relevant to the proof so these symbols can change values from one appearance to the next.

Proof of Theorem 1.1. During this proof we will write $\bar{\lambda}:=\lambda_{N(x)}, \bar{\Gamma}:=\Gamma_{N(x)}$ and $k:=j-N(x)+1$. We fix $\xi \in(1,2)$. The constant $\bar{\delta}$ that controls the size of $|\lambda|$ in the domain $\overline{\mathcal{D}}_{\lambda}$ will be the minimum of a finite number of choices that achieve bounds in generic inductive steps. The choices depend on $L$ and $\xi$. Thus, throughout the proof we will be using the following principles:

1. $\exists c:\left|\lambda_{j}\right| \leq c|\bar{\lambda}|$ for $j \geq N(x)$ by Proposition I.1.5.

2. $\left|c_{L} \lambda_{j}\right|<c$ for any $c$ because the domain for $\lambda_{0}$ is chosen after $L$ is fixed.

3. $1+c_{L}\left|\lambda_{j}\right|<\xi$ because the domain for $\lambda_{0}$ is chosen after $\xi$ is fixed.

4. $O\left(\Gamma_{j}\right) \leq c_{L}$ because $\beta_{j} \in \overline{\mathcal{D}}_{\beta}\left(\frac{1}{2}\right)$.

5. For $j \geq N(x), O\left(\Gamma_{j}\right) \leq c O(\bar{\Gamma})$ because it holds when $\left|\beta_{N(x)}\right| \leq 1$ since $\beta_{j} \in$ $\overline{\mathcal{D}}_{\beta}(1 / 2)$ and it also holds when $\left|\beta_{N(x)}\right|>1$ because then the $\beta$ recursion causes $\beta_{j}$ to grow exponentially.

Recall from Section $⿴$ that for $j \leq N(x)-1, b_{0, j}$ and $b_{2, j}$ vanish, while $b_{1, j}=L^{-2 j}$. These values will start inductive arguments at $j=N(x)-1$. The term recursion denotes the perturbative recursion (5.4) combined with the bound on the error, Proposition 6.2.

Claim 1: For $j \geq N(x),\left|b_{1, j}\right| \leq \xi^{k} L^{-2 j}, \quad\left|b_{2, j}\right| \leq O(\bar{\Gamma})|\bar{\lambda}|^{2} \xi^{k} L^{-2 j}$.

Proof. By induction using the recursion.

Claim 2: For $j \geq N(x),\left|L^{2 j} b_{1, j}-1\right| \leq \xi^{k} O\left(\bar{\Gamma}^{2}\right)|\bar{\lambda}|$.

Proof. Let $r_{k}:=\left|L^{2 j} b_{1, j}-1\right|$. Then $r_{0}=0$. By the recursion and claim $1, r_{k+1} \leq$ $r_{k}+O(\bar{\Gamma})|\bar{\lambda}| \xi^{k}$. This implies the claim.

Claim 3: For $j=N(x)-1$ let $a_{j}=0$ and for $j \geq N(x)$ let $a_{j+1}=a_{j}+L^{-2 j} \Gamma\left(\beta_{j}, L^{-2 j} x\right)$. Then

$$
\left|b_{0, j}-a_{j}\right| \leq L^{-2 N(x)} O\left(\bar{\Gamma}^{3}\right)|\bar{\lambda}|
$$

Proof. Let $r_{j}=\left|b_{0, j}-a_{j}\right|$. Then $r_{j}=0$ for $j=N(x)-1$. By the recursion and the previous claims,

$$
r_{j+1} \leq r_{j}+L^{-2 j} \xi^{k} O\left(\bar{\Gamma}^{3}\right)|\bar{\lambda}|
$$

This proves the claim. 
Recall the definition of $\hat{\beta}_{j}=\beta_{j}-\beta_{j}^{c}$ in Proposition I.1.3. Let $u_{j}:=L^{2[k-1]} \hat{\beta}_{N(x)}$. By Proposition I.1.5, $u_{j} \in \overline{\mathcal{D}}_{\beta}$.

Claim 4: For $j \geq N(x)-1$,

$$
\left|\frac{1}{1+\beta_{j}}-\frac{1}{1+u_{j}}\right|=\left|\frac{u_{j}-\beta_{j}}{\left(1+\beta_{j}\right)\left(1+u_{j}\right)}\right| \leq|\bar{\lambda}| \xi^{k} O\left(\bar{\Gamma}^{2}\right) .
$$

Proof. By Proposition I.1.3, $\left(1+\beta_{j}\right)^{-1}$ is indistinguishable from $\left(1+\hat{\beta}_{j}\right)^{-1}$ up to an error that can be absorbed in the right-hand side of our claim. By Lemma I.4.2, part (4),

$$
\hat{\beta}_{j}=u_{j} \prod_{l=N(x)}^{j-1}\left(1+O\left(\lambda_{l} O\left(\Gamma_{l}\right)\right)\right)
$$

SO

$$
\left|\hat{\beta}_{j}-u_{j}\right| \leq\left|e^{k|\bar{\lambda}| O(\bar{\Gamma})}-1\right|\left|u_{j}\right| \leq|\bar{\lambda}| O(\bar{\Gamma}) \xi^{k}\left|u_{j}\right| .
$$

The claim follows because $u /(1+u)$ is bounded for $u \in \overline{\mathcal{D}}_{\beta}$.

Claim 5: $\left|a_{N}-G_{0}\left(\beta_{\mathrm{eff}, N(x)}, x\right)\right| \leq L^{-2 N(x)}|\bar{\lambda}| O\left(\bar{\Gamma}^{2}\right)$.

Proof. By the definition of $u_{j}$ and $\beta_{\mathrm{eff}, l}=L^{-2 l} \hat{\beta}_{l}$,

$$
G_{0}\left(\beta_{\mathrm{eff}, N(x)}, x\right)=\sum L^{-2 l} \Gamma\left(u_{l}, L^{-l} x\right) .
$$

$a_{N}$ is the same expression but with $u_{l}$ replaced by $\beta_{l}$. Thus the claim is a consequence of claim 4 .

In our first paper we proved that the sequences $\left(\beta_{j}, \lambda_{j}\right)$ in Theorem 1.1 never exit the domain $\overline{\mathcal{D}}_{\beta}(1 / 2) \times \overline{\mathcal{D}}_{\lambda}$, so $M=\infty$. Recall from (4.3) that the Green's function equals $b_{0, N}$. By claims 3 and 5 ,

$$
\left|b_{0, N}-G_{0}\left(\beta_{\mathrm{eff}, N(x)}, x\right)\right| \leq L^{-2 N(x)}|\bar{\lambda}| O\left(\bar{\Gamma}^{2}\right) .
$$

By Proposition I.1.4, part (3), the right-hand side is less than $\left|G_{0}\left(\beta_{\mathrm{eff}, N(x)}, x\right)\right|$.

\section{Estimates on a Renormalization Group Step}

In this section we will prove Propositions 6.1 and 6.2 .

Let $X$ be a subset of $\Lambda$. A form $F_{X}$ is said to be localized in $X$ if it is a form on $\mathbb{C}^{X}$. Thus $F_{X}=\sum_{\alpha} F_{X, \alpha}(\phi) \psi^{\alpha}$ where $F_{X, \alpha}$ is a smooth function on $\mathbb{C}^{X}$ and $\psi^{\alpha}=$ $\prod_{x \in X} \psi_{x}^{\alpha_{x}} \bar{\psi}_{x}^{\bar{\alpha}_{x}}$. In particular, $g^{X}$ defined by $g^{X}=\prod_{x \in X} g_{x}$ is localized in $X$, when the forms $g_{x}$ are localized at single sites $\{x\}$. 
Definition 7.1. Given $h \geq 0, w>0, w^{-X}=\prod_{x \in X} w^{-1}\left(\phi_{x}\right)$, let

$$
\begin{aligned}
\left\|F_{X}\right\|_{w, h} & =\sum_{\alpha, \beta} \frac{h^{\alpha+\beta}}{\alpha ! \beta !} \sup _{\phi}\left|\partial_{\phi}^{\beta} F_{X, \alpha}\right| w^{-X}, \\
\left|F_{X}\right|_{h} & =\sum_{\alpha, \beta} \frac{h^{\alpha+\beta}}{\alpha ! \beta !}\left|\partial_{\phi}^{\beta} F_{X, \alpha}(0)\right| .
\end{aligned}
$$

These norms measure large- and small-field behaviors, respectively.

Complex Covariances and Weights: The weight $w$ is a positive function used to track decay or growth of the interaction at $\phi=\infty$. In this paper we use $w(A, \kappa):=$ $A \exp \left(-\kappa|\phi|^{2}\right)$ with $A \geq 1$ and, for tracking decay, $\kappa$ is positive. We want to see how decay is affected by convolution with Gaussian functions and forms. Let

$$
\rho_{\alpha}(\phi):=\operatorname{det}(\pi \alpha C)^{-1} e^{-\sum \alpha^{-1} \phi_{x} C_{x y}^{-1} \bar{\phi}_{y}},
$$

where $\alpha=\exp (i \theta)$ has unit modulus and positive real part and $C_{x y}$ is a positive-definite matrix with indices with $x, y \in X$. By Gaussian integration, $\left|\rho_{\alpha}\right| * w(A, \kappa)^{X}$ is bounded by $w\left(A^{\prime}, \kappa^{\prime}\right)^{X}$, where $A^{\prime}=2 A / \cos \theta$ and $\kappa^{\prime}=\kappa / 2$, if $\kappa\|C\|_{\text {operator }}$ is small enough. Thus there is a new weight $w_{\alpha C}$ such that $w_{\alpha C}^{X} \geq\left|\rho_{C}\right| * w^{X}$. Then, for any smooth function $f_{X}$,

$$
\left|\rho_{\alpha} * f_{X}\right| \leq\left|\rho_{\alpha}\right| * w^{X}\left\|w^{-X} f_{X}\right\|_{\infty}
$$

By applying this estimate also to derivatives of $f_{X}$,

$$
\left\|\rho_{\alpha} * f_{X}\right\|_{w_{\alpha C}, h} \leq\left\|f_{X}\right\|_{w, h} .
$$

We use the notation $\partial_{\psi}^{\alpha}=\prod_{x} \partial_{\psi_{x}}^{\alpha_{x}} \bar{\partial}_{\psi_{x}}^{\bar{\alpha}_{x}}$ where $\partial_{\psi_{x}}$ is a formal anti-derivative with respect to $\psi_{x}$.

Lemma 7.2. Properties of the norms:

(i) $\left\|g^{X}\right\|_{w, h} \leq\|g\|_{w, h}^{X}$, where $\|g\|_{w, h}^{X}=\prod_{x \in X}\left\|g_{x}\right\|_{w, h}$

(ii) $\left\|f_{x} g_{x}\right\|_{w, h}=\left\|f_{x}\right\|_{w_{f}, h}\left\|g_{x}\right\|_{w_{g}, h}$ when $w_{f} w_{g}=w$

(iii) $\left\|\mathcal{S} F_{X}\right\|_{\bar{w}, \mathcal{S} h} \leq\left\|F_{X}\right\|_{w, h}$ where $\bar{w} \geq \mathcal{S} w^{\left|\mathcal{G}_{1}\right|}$ and $\mathcal{S} h:=L h$

(iv) For $0 \leq h<h^{\prime},\left\|\partial_{\psi}^{\alpha} \partial_{\phi}^{\beta} F_{X}\right\|_{w, h} \leq(\alpha+\beta) !\left(h^{\prime}-h\right)^{-\alpha-\beta}\left\|F_{X}\right\|_{w, h^{\prime}}$

(v) $\left\|\mu_{C} * F_{X}\right\|_{w_{\alpha C}, h} \leq \exp \left[\sum_{x, y \in X}\left|C_{x y}\right| h^{-2}\right]\left\|F_{X}\right\|_{w, h}$

(i) - (iv) are valid when $\|\cdot\|_{*, h}$ is replaced by $|\cdot|_{h}$. 
Proof. Properties (i) - (iv) need only be proved for the $w, h$ norm because the $|\cdot|_{h}$ norm is the limit as $\kappa \rightarrow-\infty$ of the $\|\cdot\|_{w, h}$ norm, when $w=w(1, \kappa)$.

Parts (i) and (ii) are proved on page 103 and Appendix A of [BEI92]. (iii) is easy. (iv) is proved on page 104 of [BEI92]. (v) is also proved starting on page 104, noting that $(7.2)$ is (6.4) in [BEI92].

The results of this section are organized by increasing number of hypotheses. Each proposition assumes the hypotheses that have been given earlier. The important point is that each hypothesis is satisfied when: (a) $L \geq 2$ is sufficiently large; (b) $|\lambda|$ is sufficiently small depending on $L$; and (c) $h=|\lambda|^{-1 / 4}$.

The constants $c, c_{L}, c_{q}, \ldots$ that appear in hypotheses and conclusions are numbers in $(0, \infty) . \quad c_{*}$ denotes a number in $(0, \infty)$ whose value is permitted to depend on $*$, whereas $c$ is a number determined independently of all parameters $L, \lambda, \beta$ and others that appear in the theorems. These symbols are permitted to change value from one appearance to the next. Constants that always have the same value in all appearances will be denoted by a letter other than $c$.

Hypothesis $(\mu): \mu=\mu_{e^{i \theta} C}$ where $C_{x y}$ is a positive semi-definite matrix such that $C_{x y}=0$ if $|x-y|>L, \cos \theta \geq c_{1}$ and $\left|C_{x, y}\right| \leq c_{2}$.

The constants $c_{1}, c_{2}$ in this hypothesis can be chosen arbitrarily. Once they are fixed; estimates below are uniform in the choice of $\mu$. Constants appearing without qualification in hypotheses can be chosen arbitrarily.

In the following lemma, $\Delta=\Delta_{\Gamma}$ is the Laplacian defined in (5.5) with $\Gamma$ replaced by $\exp (i \theta) C$ and $\|C\|$ is the maximum of $\left|C_{x y}\right| . E_{q}(\Delta)$ is the power series $\sum_{n \leq q} \Delta^{n} / n$ ! for $\exp (\Delta)$ truncated at order $q . X$ is a subset of a $\mathcal{G}_{1}$ coset.

Hypothesis $(\mathfrak{h}): 0 \leq \mathfrak{h} \leq h$. The parameter for the small-field norm $|\cdot|_{\mathfrak{h}}$ specifies how large a "small field" can be. In most cases this is determined by the covariance $C$ (so $\mathfrak{h} \approx\|C\|^{1 / 2}$ ) but occasionally it is useful to let it be determined by the interaction $\left(\right.$ so $\left.\mathfrak{h}=h \approx \lambda^{-1 / 4}\right)$.

Lemma 7.3. $(\mathcal{S} \mu \approx \mathcal{S}):$ Let $\bar{w} \geq \mathcal{S} w_{t C}^{X}$ for $t \in[0,1]$.

$$
\begin{aligned}
& \text { (i) }\left\|\mathcal{S} \mu * g^{X}-\mathcal{S} E_{q-1}(\Delta) g^{X}\right\|_{\bar{w}, \mathcal{S} h} \leq c_{q, L} h^{-2 q}\|C\|^{q}\|g\|_{w, 2 h}^{X} \\
& \text { (ii) }\left|\mathcal{S} \mu * g^{X}-\mathcal{S} E_{q-1}(\Delta) g^{X}\right|_{\mathfrak{h}} \leq c_{q, L} h^{-2 q} \bar{w}(0)\|C\|^{q}\|g\|_{w, h}^{X}, \quad q=1,2, \ldots
\end{aligned}
$$

Proof. Let $\mu_{t}$ be $\mu$ with $e^{i \theta} C$ replaced by $t e^{i \theta} C$. Then

$$
\mathcal{S} \mu * g^{X}-\mathcal{S} E_{q-1}(\Delta) g^{X}=R_{q} \text {, with } R_{q}=\int_{0}^{t} \frac{(1-t)^{q-1}}{(q-1) !} \mathcal{S} \mu_{t} * \Delta^{q} g^{X} d t \text {. }
$$

By parts (iii) - (v) of Lemma 7.2 ,

$$
\left\|R_{q}\right\|_{\bar{w}, \mathcal{S} h} \leq c_{q, L} h^{-2 q}\|C\|^{q}\|g\|_{w, 2 h}^{X} .
$$

In part (ii), $\left|R_{q}\right|_{\mathfrak{h}}$ is bounded by $\bar{w}(0)\left\|R_{q}\right\|_{\bar{w}, \mathcal{S} h / 2}$. 
Hypothesis $(\lambda): \lambda$ lies in a sector $|\lambda| \leq c_{1} \operatorname{Re} \lambda$ in the right half of the complex plane and $|\lambda| \leq c$, where $c$ is small.

The qualification "where $c$ is small" means that each of the following results of this section may require a hypothesis $|\lambda| \leq c$, with $c$ determined in the proof.

Hypothesis $\left(h \approx \lambda^{-1 / 4}\right):|\lambda| h^{4} \in\left[c^{-1}, c\right]$. The default choice is $h=|\lambda|^{-1 / 4}$ but this assumption allows us to assume the same estimates for e.g., $2 h$.

Hypothesis $(\hat{v}, w):|\nu| \leq c_{1} h^{-2}, \hat{v}=\lambda \tau^{2}+\nu \tau+\gamma \mathcal{O}$ and $w(\phi)=e^{-a|\phi / h|^{2}}$ with $4 c_{1} \leq a \leq c_{2}$. The default choice is $a=1$ and $\nu \leq \frac{1}{2} h^{-2}$. The observable $\mathcal{O}$ is a polynomial as in (4.2) such that $\left|\gamma b_{1}\right| h^{4}+\left|\gamma b_{2}\right| h^{4} \leq c$, where $c$ is small. f

The hypothesis on the observable ensures that it is small relative to $\lambda \tau^{2}$. In principle $\gamma$ is infinitesimal because we only use the derivative with respect to $\gamma$ at zero, but it seems to be simpler to allow a finite variation of $\gamma$. The $(\hat{v}, w)$ hypothesis implies that $|\hat{v}|_{h} \leq c$.

Referring to the paragraph Complex Covariances and Weights, we find that the weight $w$ in the $(\hat{v}, w)$ hypothesis is $w(A, \kappa)$ with $A=1$ and $\kappa=a h^{-2}$. Thus $\kappa \approx|\lambda|^{1 / 2}$ is small, as required, and we can choose $\bar{w}=\mathcal{S} w\left(A^{\prime}, \kappa^{\prime}\right)^{X}$ in Lemma 7.3. Next, we can reduce to $A^{\prime}=1$ in $w\left(A^{\prime}, \kappa^{\prime}\right)$ by putting a constant $c_{L}=A^{\prime X}$ in front of the norm. Finally, the rescaling $\mathcal{S}$ maps $w\left(1, \kappa^{\prime}\right)^{X}$ into $w\left(1, L^{2} \kappa^{\prime}\right)$, which brings the decay rate $\kappa$ back to better than the original value. In particular we can choose $\bar{w}=w^{2}$ for $L>2$. To summarize: for $\lambda$ small, the rescaling more than restores the loss of decay caused by convolution so that the whole RG map will actually strengthen this parameter in the norm.

Lemma 7.4. (Integrability of $\left.e^{-\hat{v}}\right)$ : If $M$ is a polynomial form with degree $m$, then

$$
\left\|M e^{-\hat{v}}\right\|_{w, h} \leq c_{m}(h / \mathfrak{h})^{m}|M|_{\mathfrak{h}}, \quad\left|f e^{\hat{v}}\right|_{\mathfrak{h}} \leq c|f|_{\mathfrak{h}} .
$$

Proof of second estimate. $\left|f e^{\hat{v}}\right|_{\mathfrak{h}} \leq|f|_{\mathfrak{h}} e^{|\hat{v}|_{\mathfrak{h}}}$ and $|\hat{v}|_{\mathfrak{h}} \leq c|\hat{v}|_{h} \leq c$ by hypotheses.

The first estimate is reduced to the case $h=1, \lambda=c$ by scaling $\phi=h \phi^{\prime}$. See the proof of (7.4) in BEI92]. Similarly we can bound the $\exp (-\nu \tau)$ in $\exp (-\hat{v})$ for $\nu$ either positive or negative, but we must then have a weight that allows growth at infinity as in

Lemma 7.5. (Integrability of $\left.e^{\nu \tau}\right):\left\|e^{-\nu \tau}\right\|_{w^{-1}, h} \leq c$ and $|\exp (-\nu \tau)|_{\mathfrak{h}} \leq c$.

Lemma 7.6. (Interaction scales down): If $r$ is normalized, then $\|r \exp (-\hat{v})\|_{w, h} \leq$ $c L^{-6}\|r\|_{w, \mathcal{S} h / 2}$ and $|r|_{\mathfrak{h}^{\prime}} \leq c\left[\mathfrak{h}^{\prime} /\left(\mathfrak{h}-\mathfrak{h}^{\prime}\right)\right]^{6}|r|_{\mathfrak{h}}$ for $\mathfrak{h}^{\prime}<\mathfrak{h}$. More generally, if (c.f. Definition 4.1), $r_{t}=O\left(t^{p}\right)$, then $L^{-6}$ is replaced by $L^{-p}$.

\footnotetext{
${ }^{4}$ When $|x|=L$, the observable is $\gamma \phi_{0} \bar{\phi}_{x}$. But as far as estimates are concerned, the behavior is the same as that of $\frac{\gamma}{2}\left(\phi_{0} \bar{\phi}_{0}+\phi_{x} \bar{\phi}_{x}\right)$.
} 
Proof. By Taylor's theorem in $t$ applied to $r_{t}=r(t \phi)$ it suffices to estimate the $w, h$ norm of

$$
\int_{0}^{1} \frac{(1-t)^{5}}{5 !} \frac{d^{6}}{d t^{6}} r_{t} e^{-\hat{v}} d t
$$

which is bounded by $c$ times terms of the form $\sup _{t}\left\|r_{t}^{(6)} M e^{-\hat{v}}\right\|_{w, h}$ where $r^{(6)}$ denotes a sixth $\phi, \psi$ derivative of $r$ and $M$ is a monomial in $\phi, d \phi$ of degree 6 . This is less than

$$
\begin{aligned}
\sup _{t}\left\|r_{t}^{(6)}\right\|_{w_{t}, h}\left\|M e^{-\hat{v}}\right\|_{w / w_{t}, h} & =\sup _{t}\left\|r^{(6)}\right\|_{w, t h}\left\|M e^{-\hat{v}}\right\|_{w / w_{t}, h} \\
& \leq\left\|r^{(6)}\right\|_{w, h}\left\|M e^{-\hat{v}}\right\|_{w, h},
\end{aligned}
$$

where $w_{t}=w(t \phi)$, because the norm is decreasing in $w$ and $w / w_{t} \geq w \cdot r^{(6)}$ is bounded by $c(\mathcal{S} h)^{-6}$ times the $w, \mathcal{S} h / 2$ norm by Lemma 7.2 (iv) and $M e^{-\hat{v}}$ is bounded by $h^{6}$ using Lemma 7.4. The second inequality is proved by the same steps with the $\mathfrak{h}$ norms.

Lemma 7.7. (Extraction): The solution $\left(\nu^{\prime}, v^{\prime}, r^{\prime}\right)$ to

$$
e^{-\hat{v}}+\hat{r}=e^{-\nu^{\prime} \tau}\left(e^{-v^{\prime}}+r^{\prime}\right), \quad\left(v^{\prime}, r^{\prime}\right) \text { normalized }
$$

satisfies

(i) $\left|\hat{v}-\nu^{\prime} \tau-v^{\prime}\right|_{\mathfrak{h}} \leq c|\hat{r}|_{\mathfrak{h}}$

(ii) $\left|\nu^{\prime}-\nu\right| \mathfrak{h}^{2}+\left|\lambda^{\prime}-\lambda\right| \mathfrak{h}^{4} \leq c|\hat{r}|_{\mathfrak{h}}$ where $\gamma=0$ in $\hat{r}$

(iii) $\left\|r^{\prime}\right\|_{w, h} \leq c\|\hat{r}\|_{w^{2}, h}$

(iv) $\left|r^{\prime}\right|_{\mathfrak{h}} \leq c|\hat{r}|_{\mathfrak{h}}$

provided that for each estimate the right-hand side is less than a constant $c$ which is small.

Proof of $(i)$. Define $|\cdot|_{\mathfrak{h}}^{(p)}$ by truncating the sum in the definition of $|\cdot|_{\mathfrak{h}}$ so that only derivatives of order $p$ or less are included. This seminorm satisfies $\left|F_{1} F_{2}\right|_{\mathfrak{h}}^{(p)} \leq$ $\left|F_{1}\right|_{\mathfrak{h}}^{(p)}\left|F_{2}\right|_{\mathfrak{h}}^{(p)}$. Rewrite the $\left(\nu^{\prime}, v^{\prime}, r^{\prime}\right)$ equation,

$$
\hat{v}-\nu^{\prime} \tau-v^{\prime}=\ln \left(1+e^{\hat{v}} \hat{r}-e^{\hat{v}-\nu^{\prime} \tau} r^{\prime}\right)
$$

Expand the ln, take the $|\cdot|_{\mathfrak{h}}^{(p)}$ with $p=4$ :

$$
\left|\hat{v}-\nu^{\prime} \tau-v^{\prime}\right|_{\mathfrak{h}}^{(p)} \leq \sum_{j} \frac{x^{j}}{j}, x=\left|e^{\hat{v}} \hat{r}\right|_{\mathfrak{h}}^{(p)}+\left|e^{\hat{v}-\nu^{\prime} \tau}\right|_{\mathfrak{h}}^{(p)}\left|r^{\prime}\right|_{\mathfrak{h}}^{(p)}
$$


It suffices to prove the estimate for $p=4$ because $\left|\hat{v}-\nu^{\prime} \tau-v^{\prime}\right|_{\mathfrak{h}}^{(4)}=\left|\hat{v}-\nu^{\prime} \tau-v^{\prime}\right|_{\mathfrak{h}}$ whereas $|\hat{r}|_{\mathfrak{h}}^{(p)} \leq|\hat{r}|_{\mathfrak{h}} . \quad x=\left|e^{\hat{v}} \hat{r}\right|_{\mathfrak{h}}^{(p)}$ because the first five derivatives of $r^{\prime}$ are zero by normalization. Also, $\left|e^{\hat{v}} \hat{r}\right|_{\mathfrak{h}}^{(p)} \leq c|\hat{r}|_{\mathfrak{h}}$ as in the proof of Lemma 7.4. By hypothesis, the sum is dominated by the first term so that

$$
\left|\hat{v}-\nu^{\prime} \tau-v^{\prime}\right|_{\mathfrak{h}}^{(p)} \leq c|\hat{r}|_{\mathfrak{h}} .
$$

Proof of (ii). Bound the left-hand side by $c\left|\hat{v}-\nu^{\prime} \tau-v^{\prime}\right|_{\mathfrak{h}}$ and use part (i).

Proof of (iii). Let

$$
r^{\prime}(\alpha)=e^{\nu^{\prime} \tau}\left(e^{-\hat{v}}+\alpha \hat{r}\right)-e^{-v^{\prime}(\alpha)}
$$

be the solution when $\hat{r}$ is replaced by $\alpha \hat{r}$. For $\alpha$ in the disk $\|\alpha \hat{r}\|_{w^{2}, h} \leq c_{1}$, with $c_{1}$ small, we show that $\left\|r^{\prime}(\alpha)\right\|_{w, h}$ is bounded by $c$. First, $\exp \left(\nu^{\prime} \tau-\hat{v}\right)$ and $\exp \left(-v^{\prime}\right)$ satisfy a $(\hat{v}, w)$ hypothesis by (a) parts (ii) and (i) with $\mathfrak{h}=h$, and (b) $|\hat{r}|_{h} \leq\|\hat{r}\|_{w^{2}, h}$. Second,

$$
\left\|\exp \left(\nu^{\prime} \tau\right)(\alpha \hat{r})\right\|_{w, h} \leq\left\|\exp \left(\nu^{\prime} \tau\right)\right\|_{w^{-1}, h}\|\alpha \hat{r}\|_{w^{2}, h} \leq c,
$$

using Lemma 7.5. Since $r^{\prime}(\alpha)$ vanishes at $\alpha=0$ we have, by analyticity in $\alpha$,

$$
\left\|r^{\prime}\right\|_{w, h} \leq c\|\hat{r}\|_{w^{2}, h}
$$

Proof of (iv). By taking the norm of (7.5),

$$
\left|r^{\prime}(\alpha)\right|_{\mathfrak{h}} \leq e^{\left|\nu^{\prime} \tau\right|_{\mathfrak{h}}}\left(e^{|\hat{v}|_{\mathfrak{h}}}+|\alpha \hat{r}|_{\mathfrak{h}}\right)+e^{\left|v^{\prime}\right|_{\mathfrak{h}}} \leq c
$$

Therefore, as in part (iii), $\left|r^{\prime}\right|_{\mathfrak{h}} \leq c|\hat{r}|_{\mathfrak{h}}$.

Define

$$
r_{\text {main }}=\text { constant and linear in } \gamma \text { part of } \mathcal{S} \mu_{C} *\left(e^{-v}\right)^{\mathcal{G}_{1}}-e^{-\hat{v}} \text {, }
$$

with $\hat{v}=\tilde{v}+\tilde{\nu} \tau$ as in Proposition 5.1. The right-hand side is expanded in a series in powers of $\gamma$ and truncated at order $\gamma$. For computing the observable, we only need the derivative in $\gamma$ at zero so there is no loss in such truncation.

Lemma 7.8. (Remainder after perturbation theory is small in norm): Suppose that $\sum_{y} C_{x y}=0$ and $|\mathfrak{h}| \leq c_{L}\|C\|^{\frac{1}{2}}$. Then

$$
\left\|r_{\text {main }}\right\|_{w, h} \leq c_{L}\|C\|^{2}|\lambda|, \quad\left|r_{\text {main }}\right|_{\mathfrak{h}} \leq c_{L}\|C\|^{3}|\lambda|^{3} .
$$

The main obstacle to proving this lemma is the "large field problem" described at the end of Section 5. Using the notation of Lemma 5.3, let $\hat{V}_{t}=-V_{t}+Q_{t}$ with $Q$ linearized in $\gamma$ and let $E_{q}(A)=\sum_{j=0}^{q} A^{q} / q$ ! denote the exponential truncated at order $q$. We solve the large field problem by splitting $\hat{V}_{t}=\bar{J}_{t}+\underline{J}_{t}$ and using the approximation $\exp \left(\underline{J}_{t}\right) E_{q}\left(\bar{J}_{t}\right)$ in place of $\exp \left(\hat{V}_{t}\right)$. $\bar{J}_{t}$ will contain the polynomial of degree six which would ruin integrability if fully exponentiated. 
$Q_{t}$ is a sum of terms of the form $P_{x} C_{x, y}^{j} P_{y}+O(\gamma)$, where $P_{x}$ is a polynomial in $\phi_{x}, \psi_{x}$ and conjugates. Let $\underline{\mathrm{Q}}_{t}$ be the same sum with $P_{y}$ replaced by $P_{x}$. The degree 6 term that seems to be in $\underline{\mathrm{Q}}_{t}$ is actually zero because $\sum_{y} C_{x y}=0$. We choose $\underline{J}_{t}=-V_{t}+\underline{\mathrm{Q}}_{t}$. Then $\bar{J}_{t}=Q_{t}-\underline{Q}_{t}$ has the property that $\mathcal{S} \bar{J}_{t}=0$ and

$$
\hat{v}=\mathcal{S} \hat{V}_{t=1}=-\mathcal{S} \underline{J}_{t=1}
$$

Since $\lambda$ is small we can assume the same $(\hat{v}, w)$ hypothesis for $v$ and $\hat{v}$.

Note that $\underline{J}_{t}$ contains the $\tau^{2}$ terms. We keep them in a standard exponent because $\exp \left(-\lambda \tau^{2}\right)$ is useful for suppressing large values of $\phi$, unlike $E_{q}\left(-\lambda \tau^{2}\right)$.

Proof of Lemma 7.8. Equation (7.7) implies that at $t=1, \mathcal{S} \exp \left(\underline{J}_{t}\right) E_{q}\left(\bar{J}_{t}\right)=e^{-\hat{v}}$. Recall from Section 5 the Duhamel formula

$$
r_{\text {main }}=\mathcal{S} \int_{0}^{1} d t \mu_{(1-t)} * \mathcal{L} e^{\underline{J}_{t}} E_{q}\left(\bar{J}_{t}\right) .
$$

By a calculation based on the differentiation rule $D E_{q}(\bar{J})=E_{q-1}(\bar{J}) D \bar{J}$,

$$
\begin{gathered}
\mathcal{L} e^{\underline{J}} E_{q}(\bar{J})=e^{\underline{J}} E_{q-1}(\bar{J})\left[\mathcal{L} \hat{V}-\frac{1}{2} \hat{V} \overleftrightarrow{\Delta} \hat{V}\right]+ \\
\frac{1}{q !} e^{-} \bar{J}^{q}\left[\mathcal{L} \underline{J}-\frac{1}{2} \underline{J} \stackrel{\leftrightarrow}{\Delta} \underline{J}\right]+\frac{1}{2(q-1) !} e^{-} \bar{J}^{q-1} \bar{J} \overleftrightarrow{\Delta} \bar{J}
\end{gathered}
$$

We have omitted $t$ subscripts. We choose $q=2$. As in (5.7),

$$
\begin{aligned}
\mathcal{L} e^{\underline{J}} E_{2}(\bar{J}) & =e^{\underline{J}} E_{1}(\bar{J})\left[Q \overleftrightarrow{\Delta} V-\frac{1}{2} Q \stackrel{\leftrightarrow}{\Delta} Q\right]+ \\
& \frac{1}{2} e^{-} \bar{J}^{2}\left[\mathcal{L} \underline{J}-\frac{1}{2} \underline{J} \stackrel{\leftrightarrow}{J}\right]+\frac{1}{2} e^{-} \bar{J} \bar{J} \stackrel{\leftrightarrow}{\Delta} \bar{J}
\end{aligned}
$$

Consider the term $\exp (\underline{J}) E_{1}(\bar{J}) Q_{t} \stackrel{\leftrightarrow}{\Delta} V_{t}$ in $(\underline{7.10})$. Since $E_{1}(\bar{J})=1+\bar{J}$ it splits into two terms, one of which is $F:=\exp (\underline{J}) Q_{t} \stackrel{\leftrightarrow}{\Delta} V_{t}$. F is a sum of products of terms of the form $\left(M e^{-v}\right)^{\mathcal{G}_{1}}$ as in Lemma 7.4 because there are three sites in $\mathcal{G}_{1}$ where there are monomials corresponding to the three factors $v_{x}, v_{y}, v_{z}$ in $F$. If we consider the $\lambda \tau^{2}$ parts of $v$ then we find that the total degree of the monomials is $3 \times 4-4=8$. The -4 is because $F$ contains two $\overleftrightarrow{\Delta}$, one explicit and one in $Q_{t}$. Associated with these two $\overleftrightarrow{\Delta}$ are $C_{x, y}$ and $C_{y, z}$. There is also $\lambda^{3}$. Therefore, by Lemmas 7.2 - 7.4. $\left\|\left(M e^{-v}\right)^{\mathcal{G}_{1}}\right\|_{w, h}$ is bounded by $c_{L} h^{8}|\lambda|^{3}\|C\|^{2}=c_{L}|\lambda|\|C\|^{2}$. The $c_{L}$ includes factors $L^{4}=\left|\mathcal{G}_{1}\right|$ from sums over $x, y, z$.

The other term in $\exp (\underline{J}) E_{1}(\bar{J}) Q_{t} \stackrel{\leftrightarrow}{\Delta} V_{t}$ is $\bar{J} F$. This is smaller by an additional factor $c_{L} h^{6}|\lambda|^{2}\|C\|^{2}$ by the same methods. In fact, all of the terms in (7.8.7.10) can be estimated by this procedure by $c_{L}|\lambda|\|C\|^{2}$. The $O(\gamma)$ terms may be absorbed into these bounds because, according to the $(\hat{v}, w)$ hypothesis, $|\gamma b| \leq c|\lambda|$. 
The $|\cdot|_{\mathfrak{h}}$ norm is estimated in the same way but taking into account the hypothesis on $\mathfrak{h}$, (i) $|\lambda|^{3} \mathfrak{h}^{p} \leq c_{L, p}|\lambda|^{3}$ and (ii) if $M$ is a monomial of order $2 p$ then $|M|_{\mathfrak{h}} \leq c \mathfrak{h}^{2 p} \leq$ $c_{L, p}\|C\|^{p}$. There are at least two factors of $C$ in every term and for terms with only two at least one more is contributed by (ii).

Hypothesis ( $\lambda, r$ small depending on $L):|\lambda|,\|r\|_{w, h}$ and $(h / \mathfrak{h})^{4}|r|_{\mathfrak{h}} \leq c_{L}$ where $c_{L}$ is determined in the proof of the next theorem.

Proposition 7.9. Let $v$ be given by $(\sqrt{4.9}), h, h^{\prime}=|\lambda|^{-1 / 4},\left|\lambda^{\prime}\right|^{-1 / 4}$ and $c_{L} \sqrt{\|C\|} \geq \mathfrak{h} \geq$ $\mathfrak{h}^{\prime} \geq L \sqrt{\|C\|}$. Then the map from $(v, r)$ to $\left(v^{\prime}, r^{\prime}, \nu^{\prime}\right)$ defined by

$$
\mathcal{S} \mu *\left(e^{-v}+r\right)^{\mathcal{G}_{1}}=e^{-\nu^{\prime} \tau}\left(e^{-v^{\prime}}+r^{\prime}\right),
$$

with $v^{\prime}, r^{\prime}$ normalized, satisfies

$$
\begin{gathered}
\text { (i) }\left\|r^{\prime}\right\|_{w, h^{\prime}} \leq c_{L}|\lambda|\|C\|^{2}+c L^{-2}\|r\|_{w, h} \\
\begin{array}{c}
\text { (ii) }\left|r^{\prime}\right|_{\mathfrak{h}^{\prime}} \leq c_{L}|\lambda|^{3}\|C\|^{3}+c L^{-2}\left(\mathfrak{h}^{\prime} / \mathfrak{h}\right)^{6}|r|_{\mathfrak{h}}+c_{L}\|C\|^{5} h^{-10}\|r\|_{w, h} \\
(i i i)\left|\beta^{\prime}-\tilde{\beta}\right| \mathfrak{h}^{2}+\left|\lambda^{\prime}-\tilde{\lambda}\right| \mathfrak{h}^{4} \leq c_{L}|\lambda|^{3}\|C\|^{3}+c L^{-2}\left(\mathfrak{h}^{\prime} / \mathfrak{h}\right)^{6}|r|_{\mathfrak{h}} \\
+c_{L} h^{-10}\|C\|^{5}\|r\|_{w, h} .
\end{array}
\end{gathered}
$$

Proof of (i) and (ii). By solving $\mathbb{T}_{\beta}(\exp (-v)+r)^{\mathcal{G}_{1}}=\exp (-\hat{v})+\hat{r}$ for $\hat{r}$,

$$
\begin{aligned}
\hat{r} & =r_{\text {main }}+\mathcal{S} E_{q-1}(\Delta) \sum_{x \in \mathcal{G}_{1}}\left(e^{-v}\right)^{\mathcal{G}_{1} \backslash\{x\}} r_{x} \\
& +\mathcal{S} E_{q-1}(\Delta) \sum_{X \subset \mathcal{G}_{1},|X|>1}\left(e^{-v}\right)^{\mathcal{G}_{1} \backslash X} r^{X} \\
& +\left(\mathbb{T}_{\beta}-\mathcal{S} E_{q-1}(\Delta)\right) \sum_{X \subset \mathcal{G}_{1}}\left(e^{-v}\right)^{\mathcal{G}_{1} \backslash X} r^{X} .
\end{aligned}
$$

First term in (7.11): By Lemma $7.8\left\|r_{\text {main }}\right\|_{w^{2}, h} \leq c_{L}\|C\|^{2}|\lambda|$.

Second term in (7.11): We choose $q=1$. Let $X=\mathcal{G}_{1} \backslash\{x\} \cdot \mathcal{S}(\exp (-v))^{X}$ equals $\exp \left(-v_{\text {new }}\right)$ where $v_{\text {new }}$ still satisfies the $(\hat{v}, w)$ hypothesis?. By Lemma 7.6, Lemma 7.2 part (iii) and $w^{2} \geq \mathcal{S} w^{\mathcal{G}_{1}}$

$$
\left\|\sum_{x \in \mathcal{G}_{1}} \mathcal{S}\left(e^{-v}\right)^{X} \mathcal{S} r_{x}\right\|_{w^{2}, 2 h} \leq c L^{4} L^{-6}\|\mathcal{S} r\|_{w^{2}, \mathcal{S} h} \leq c L^{-2}\|r\|_{w, h}
$$

Third term in (7.11): By Lemmas 7.2 and 7.4, $\|$ third term $\left\|_{w^{2}, 2 h} \leq c_{L}\right\| r \|_{w, h}^{2}$.

Fourth term in (7.11): By Lemma 7.3, with $\bar{w}=w^{2}$ as explained below the $(\hat{v}, w)$ hypothesis, followed by Lemmas 7.2 and 7.4, $\|$ fourth term $\left\|_{w^{2}, 2 h} \leq h^{-2}\right\| r \|_{w, h}$ because

\footnotetext{
${ }^{5}$ because it equals $\lambda_{\text {new }} \tau^{2}+$ observable with $\lambda_{\text {new }}=\lambda\left(1-L^{-4}\right)$.
} 
there are less than $c_{L}$ terms in the sum over $X$ and each has one or more factors of $r$ and $q=1$.

Therefore

$$
\|\hat{r}\|_{w^{2}, 2 h} \leq c_{L}|\lambda|\|C\|^{2}+c L^{-2}\|r\|_{w, h},
$$

where we used the hypotheses $\left(r\right.$ and $h^{-2}=\sqrt{|\lambda|}$ small depending on $\left.L\right)$ to put the estimates for the third and fourth terms inside $c L^{-2}\|r\|_{w, h}$.

To estimate the $\mathfrak{h}^{\prime}$ norm, we use (7.11) with $q=5$. Consider the second term in (7.11). Let

$$
F=\sum_{x \in \mathcal{G}_{1}}\left(e^{-v}\right)^{\mathcal{G}_{1} \backslash\{x\}} r_{x}
$$

Note that $F_{t}=O\left(t^{6}\right)$ is normalized, using the subscript $t$ notation of Definition 4.1. However, $\left(\Delta^{n} F\right)_{t}=O\left(t^{6-2 n}\right)$. By Lemma 7.6, with $\mathfrak{h}$ replaced by $L \mathfrak{h} / 2$, and Lemma 7.2 part (iii)

$$
\left|\mathcal{S} \Delta^{n} F\right|_{\mathfrak{h}^{\prime}} \leq c\left[\mathfrak{h}^{\prime} /(L \mathfrak{h})\right]^{6-2 n}\left|\Delta^{n} F\right|_{\mathfrak{h} / 2}
$$

for $n=0,1,2$. When $n=3,4,5$ we still have this bound (by Lemma 7.2 (iii)) because $\mathfrak{h}^{\prime} /(L \mathfrak{h}) \leq 1$. By Lemma 7.4, $|\exp (-v)|_{\mathfrak{h}} \leq 1+L^{-4}$ for $|\lambda|<c_{L}$. Therefore, by Lemma 7.2,

$$
\left|\mathcal{S} \Delta^{n} F\right|_{\mathfrak{h}^{\prime}} \leq c L^{4}\left[\mathfrak{h}^{\prime} /(L \mathfrak{h})\right]^{6-2 n}\left[\|C\| \mathfrak{h}^{-2}\right]^{n}|r|_{\mathfrak{h}}
$$

and then, by $\mathfrak{h}^{\prime} \geq L \sqrt{\|C\|}$,

$$
\mid \text { second term in }\left.(\overline{7.11})\right|_{\mathfrak{h}^{\prime}} \leq c L^{-2}\left[\mathfrak{h}^{\prime} / \mathfrak{h}\right]^{6}|r|_{\mathfrak{h}} \text {. }
$$

The third term in (7.11) is absorbed into the same bound by using the same argument. There are merely more factors of $r$ which make it much smaller by the hypothesis on $|r|_{\mathfrak{h}}$. The fourth term in $(7.11)$ is bounded by Lemma 7.3 so that

$$
|\hat{r}|_{\mathfrak{h}^{\prime}} \leq c_{L}|\lambda|^{3}\|C\|^{3}+c L^{-2}\left(\mathfrak{h}^{\prime} / \mathfrak{h}\right)^{6}|r|_{\mathfrak{h}}+c_{L}\|C\|^{5} h^{-10}\|r\|_{w, h}
$$

The proof of (i) and (ii) is completed by applying Lemma 7.7 to (7.13, 7.14).

Proof of (iii). Immediate consequence of Lemma 7.7 and (7.13,7.14).

Proof of Proposition 6.1. Recall that the finite volume Green's function $G_{\lambda_{0}}^{\Lambda_{0}}\left(\beta_{0}, x\right)$ has been expressed as an integral, (4.3). This integral is convergent for all $\beta_{0} \in \mathbb{C}$, provided the real part of $\lambda_{0}$ is positive, which is the case for $\lambda_{0} \in \overline{\mathcal{D}}_{\lambda}$. In this case we can also define $\left(\beta_{j}, \lambda_{j}, r_{j}\right)$ by conglomerating the $j \mathrm{RG}$ steps into one giant step (for $\mathrm{RG}$ ), with covariance equal to the sum of all the covariances of the individual RG steps, and applying it to the initial interaction. The big RG step is a convolution of a Gaussian 
into an interaction that decays faster than any Gaussian so it is convergent and the result defines $\left(\beta_{j}, \lambda_{j}, r_{j}\right)$. Furthermore, $r_{j}$ is entire in $\phi$. The sequence $\left(\beta_{j}, \lambda_{j}, r_{j}\right)$ is defined for all $j$ by the remark below (4.1).

In order to obtain useful estimates on the sequence, we rotate the contours of integration of each $\phi_{x}$ (both real and imaginary parts). For any $|\theta| \leq b_{\beta}+b_{\lambda} / 4+$ $9 \epsilon / 8-\pi / 2$ we may rotate $\phi_{x} \rightarrow \exp (i \theta / 2) \phi_{x}$ and $\bar{\phi}_{x} \rightarrow \exp (i \theta / 2) \bar{\phi}_{x}$. Likewise $\psi_{x}=$ $(2 \pi i)^{-1 / 2} d \phi_{x}$ and $\bar{\psi}_{x}=(2 \pi i)^{-1 / 2} d \bar{\phi}_{x}$ pick up factors $\exp (i \theta / 2)$. The effect of the rotation is to replace $\beta, \lambda, A$ with

$$
\beta(\theta)=e^{i \theta} \beta, \lambda(\theta)=e^{2 i \theta} \lambda, \quad A(\theta)=e^{i \theta} A .
$$

Since the covariance $A_{x y}^{-1}=U^{\Lambda}(\beta, x-y)$ is determined by $\Gamma$ through (3.4), the rotation of $A$ is equivalent to an anti-rotation $\Gamma(\beta) \rightarrow \Gamma(\theta)=\Gamma(\beta) \exp (-i \theta)$. Recalling the discussion of $b_{\beta}, b_{\lambda}$ below (I.1.15), the above condition on $\theta$ is sufficient to ensure that $|\arg \lambda(\theta)|<\pi / 2-\epsilon / 4$ for any $|\arg \lambda|<b_{\lambda}+\epsilon$, since by assumption,

$$
2\left(b_{\beta}+\epsilon\right)+\frac{3}{2}\left(b_{\lambda}+\epsilon\right)<\frac{3}{2} \pi .
$$

Hence the integrals remain rapidly convergent at $\infty$ and there is no contribution from the contours at $\infty$. We obtain the functional equation

$$
G_{\lambda, \Gamma}^{\Lambda}(\beta, x)=G_{\lambda(\theta), \Gamma(\theta)}^{\Lambda}(\beta(\theta), x) e^{i \theta}
$$

The sequence $\left(\beta_{j}, \lambda_{j}, r_{j}\right)$ transforms to $\left(\beta_{j}(\theta), \lambda_{j}(\theta), r_{j}(\theta)\right)$, where $\beta_{j}(\theta)=\beta_{j} \exp (i \theta)$, $\lambda_{j}(\theta)=\lambda_{j} \exp (2 i \theta)$, and $r_{j}(\theta, \phi)=r_{j}(\phi \exp (i \theta))$. It is evident that $\beta_{j}, \lambda_{j}$ transform in the same manner as $\beta, \lambda$ because shifts in the coupling were defined in Section 1 as derivatives of functions of $\phi$, now $\phi \exp (i \theta / 2)$ and each derivative introduces a factor $\exp (i \theta / 2)$.

Define a decomposition $\overline{\mathcal{D}}_{\beta}\left(\frac{1}{2}\right)=H^{+} \cup H^{-}$of the $\beta$ domain into two overlapping fattened sectors. Here

$$
H^{ \pm}=\left\{\beta \neq 0:|\arg \beta \mp \theta|<\frac{\pi}{2}-\frac{\epsilon}{8}\right\}+\mathcal{B}\left(\frac{1}{2}\right),
$$

where $\theta=b_{\beta}+b_{\lambda} / 4+9 \epsilon / 8-\pi / 2$.

Claim 1: Suppose that $\beta_{0}$ is in $H_{-}$and $\lambda_{0}$ is in $\overline{\mathcal{D}}_{\lambda}$. Let $\mathfrak{h}_{j}=L\left\|\Gamma\left(\beta_{j-1}\right)\right\|^{1 / 2}$. For $L$ sufficiently large, there is a constant $k_{L}$ such that

$$
\begin{aligned}
& \left\|r_{j}(\theta)\right\|_{w, h_{j}} \leq k_{L}\left|\lambda_{j-1}\right|\left\|\Gamma\left(\beta_{j-1}\right)\right\|^{\frac{1}{2}} \\
& \left|r_{j}(\theta)\right|_{\mathfrak{h}_{j}} \leq k_{L}\left|\lambda_{j-1}\right|^{3}\left\|\Gamma\left(\beta_{j-1}\right)\right\|^{3},
\end{aligned}
$$

for all $j \geq 1$ such that $\left(\beta_{j-1}, \lambda_{j-1}\right)$ lies in $H_{-} \times \overline{\mathcal{D}}_{\lambda}$. The analogous claim with $H_{-}$ replaced by $H_{+}$and $r_{j}(\theta)$ replaced by $r_{j}(-\theta)$ is also true. 
Proof by induction. Since $\lambda_{j-1} \in \overline{\mathcal{D}}_{\lambda}$, we have as above that $\left|\arg \lambda_{j-1}(\theta)\right|<$ $\pi / 2-\epsilon / 8$ and so the $\lambda$ hypothesis is valid for $\lambda_{j}(\theta)$. The covariance is

$$
\Gamma(\theta)=\Gamma\left(\beta_{j-1}\right) e^{-i \theta}=\frac{e^{-i \theta}}{1+\beta_{j-1}} \Gamma(0)=\frac{e^{-i\left(\theta+\arg \left(1+\beta_{j-1}\right)\right)}}{\left|1+\beta_{j-1}\right|} \Gamma(0),
$$

and we verify the $\mu$ hypothesis. First we observe that $\Gamma(0)$ is positive semi-definite. Second, since $b_{\beta}+\epsilon<3 \pi / 4$, we have that $\left|1+\beta_{j-1}\right|>(\sqrt{2}-1) / 2$. Third, our definition of $H^{-}$guarantees that $\left|\theta+\arg \left(1+\beta_{j-1}\right)\right|<\pi / 2-\epsilon / 8$.

As a result, Proposition 7.9 is applicable to the rotated parameters. By part (ii),

$$
\left|r_{j+1}(\theta)\right|_{\mathfrak{h}_{j+1}} \leq \frac{1}{2} k_{L}\left|\lambda_{j}\right|^{3}\left\|\Gamma\left(\beta_{j}\right)\right\|^{3}+\frac{1}{4}\left(\mathfrak{h}_{j+1} / \mathfrak{h}_{j}\right)^{6}\left|r_{j}(\theta)\right|_{\mathfrak{h}_{j}}+c_{L}\left\|\Gamma\left(\beta_{j}\right)\right\|^{5} h^{-10}\left\|r_{j}(\theta)\right\|_{w, h_{j}} .
$$

We made a $j$ independent choice of $L$ large to get the second term in this particular form. The first term has the constant $k_{L}$ because we choose $k_{L}$ in the inductive hypothesis to make it so. This bound proves the second inequality of claim 1 for $j=1$ because $r_{0}=0$. In the right-hand side, substitute the inductive assumptions (7.16) to obtain

$$
\left|r_{j+1}(\theta)\right|_{\mathfrak{h}_{j+1}} \leq k_{L}\left(\frac{1}{2}\left|\lambda_{j}\right|^{3}\left\|\Gamma\left(\beta_{j}\right)\right\|^{3}+\frac{3}{8}\left|\lambda_{j-1}\right|^{3}\left\|\Gamma\left(\beta_{j}\right)\right\|^{3}\right)
$$

where $c_{L}\left\|\Gamma\left(\beta_{j}\right)\right\|^{5} h^{-10}\left\|r_{j}(\theta)\right\|_{w, h_{j}}$ went into the second term by a $j$ independent choice of $\overline{\mathcal{D}}_{\lambda}$ so that $|\lambda|$ is small, making $h$ large and consequently $h_{j}^{-10} \ll\left|\lambda_{j}\right|^{2}$. By the $\lambda$ recursion $\frac{3}{8}\left|\lambda_{j-1}\right| \leq \frac{4}{8}\left|\lambda_{j}\right|$. Thus we have advanced $j$ to $j+1$ in the second estimate estimate of claim 1. The advance of $j$ to $j+1$ in the first estimate is proved similarly. But first observe that there exists an $L$-independent constant $k$ such that

$$
\frac{\left\|\Gamma\left(\beta_{j-1}\right)\right\|}{\left\|\Gamma\left(\beta_{j}\right)\right\|}=\left|\frac{1+\beta_{j}}{1+\beta_{j-1}}\right|=\left|\frac{1+\beta_{j}}{1+L^{-2} \beta_{j}+O\left(\lambda_{j-1}\right)}\right| \leq k^{2} L^{2}
$$

for all $\beta_{j} \in \overline{\mathcal{D}}_{\beta}(1 / 2)$. (The relation between $\beta_{j-1}$ and $\beta_{j}$ follows from Proposition 7.9 (iii) and the inductive assumption (7.16)). Hence, by Proposition 7.9 (i) and (7.16), we have

$$
\left\|r_{j+1}(\theta)\right\|_{w, h_{j+1}} \leq k_{L}\left\|\Gamma\left(\beta_{j}\right)\right\|^{\frac{1}{2}}\left(\frac{1}{2}\left|\lambda_{j}\right|+c L^{-2} k L\left|\lambda_{j-1}\right|\right),
$$

so we may choose $L>4 c k$ to complete the induction.

For later use we observe that if, in the above argument, we use (7.13, 7.14) in place of Proposition 7.9 (i) and (ii), we obtain

Claim 1': (7.16) holds when $r_{j+1}$ is replaced by $\hat{r}_{j}$.

Claim 2: Let $\left(\beta_{j}, \lambda_{j}\right)$ with $j=0,1, \ldots, M-1$ be an RG sequence in $\overline{\mathcal{D}}_{\beta}(1 / 2) \times \overline{\mathcal{D}}_{\lambda}$. If $\beta_{j}$ lies in $H_{-} \backslash H_{+}$, then $\beta_{j+1}$ is not in $H_{+}$.

The idea is that the factor $L^{2}$ in the $\beta$ recursion causes a large expansion in $\beta$ in a direction that takes $\beta_{j}$ further from $H_{+}$. There are other terms in the $\beta$ recursion, but they are small by claim 1 . 
Proof. By the hypothesis, $\operatorname{Re} \beta_{j}(-\theta-\epsilon / 8) \leq-\frac{1}{4}$. Therefore, $\operatorname{Re} L^{2} \beta_{j}(-\theta-\epsilon / 8) \leq$ $-\frac{1}{4} L^{2}$, so no $\beta$ within distance one of $L^{2} \beta_{j}$ can be in $H_{+}$. On the other hand, by claim 1 and part (iii) of Proposition 7.9, $\left|\beta_{j+1}(\theta)-\tilde{\beta}_{j+1}(\theta)\right| \ll 1$. Therefore $\left|\beta_{j+1}-L^{2} \beta_{j}\right| \ll 1$. Consequently $\beta_{j+1}$ is not in $H_{+}$.

By claim 2, either the whole sequence $\left(\beta_{j}, \lambda_{j}\right)$ with $j=0,1, \ldots, M-1$ lies in $H_{-}$ or it lies in $H_{+}$or in both. Therefore Proposition 6.1 follows from claim 1 applied with either $H_{+}$or $H_{-}$, Proposition 7.9 (iii), along with (5.3).

Remark: The functional equation (7.15) provides an analytic continuation of $G_{\lambda}$ into the larger domain $|2 \arg \beta-(3 / 2) \arg \lambda|<3 \pi / 2,|\arg \beta|<\pi,|\arg \lambda|<\pi$, even though the defining integral fails to converge for $|\arg \lambda|>\pi / 2$. However, to obtain the end-to-end distance from $G_{\lambda}$, we require $b_{\beta}>\pi / 2$ and so $b_{\lambda}<\pi / 3$.

Proposition 6.2 involves derivatives with respect to the observable parameter $\gamma$ at $\gamma=0$. Derivatives at zero will be denoted by $\gamma$ subscripts as in $v_{\gamma}$ and $r_{\gamma}$. We will repeatedly use four principles: (1) distinguishing partial dependences on $\gamma$ by subscripts on $\gamma ;(2)$ the total $\gamma$ derivative is the sum of the partial derivatives with respect to partial dependences; (3) Functions of $\gamma$ may be replaced by linearizations; and (4) Cauchy estimates on derivatives. These are illustrated in detail in the proof of Corollary 7.10 and are subsequently used without comment.

Corollary 7.10. (to Lemma 7.7)

(i) $\left|\hat{v}_{\gamma}-v_{\gamma}^{\prime}\right|_{\mathfrak{h}} \leq c\left(|\hat{r}|_{\mathfrak{h}}\left|\hat{v}_{\gamma}\right|_{\mathfrak{h}}+\left|\hat{r}_{\gamma}\right|_{\mathfrak{h}}\right)$

(ii) $\left|r_{\gamma}^{\prime}\right|_{\mathfrak{h}} \leq c\left(\left|\hat{r}_{\gamma}\right|_{\mathfrak{h}}+|\hat{r}|_{\mathfrak{h}}\left|\hat{v}_{\gamma}\right|_{\mathfrak{h}}\right)$

(iii) $\left\|r_{\gamma}^{\prime}\right\|_{w, h} \leq c\left(\left\|\hat{r}_{\gamma}\right\|_{w^{2}, h}+\|\hat{r}\|_{w^{2}, h}\left|\hat{v}_{\gamma}\right|_{h}\right)$

Proof of (i) . Firstly, $\hat{v}-\nu^{\prime} \tau-v^{\prime}$ is a function of $\gamma_{\hat{v}}$ when $\hat{v}$ is replaced by $\hat{v}+\gamma_{\hat{v}} \hat{v}_{\gamma}$. It is $\mathfrak{h}$ norm analytic and bounded by $c$ uniformly in the disk $\left|\gamma_{\hat{v}} \hat{v}_{\gamma}\right|_{\mathfrak{h}} \leq c$. By the Cauchy formula for the derivative at $\gamma=0$ and Lemma 7.7 part $(\mathrm{i}),\left|\left(\hat{v}-\nu^{\prime} \tau-v^{\prime}\right)_{\gamma_{\hat{v}}}\right|_{\mathfrak{h}} \leq c|\hat{r}|_{\mathfrak{h}}\left|\hat{v}_{\gamma}\right|_{\mathfrak{h}}$. Secondly, $\hat{v}-\nu^{\prime} \tau-v^{\prime}$ is a function of $\gamma_{\hat{r}}$ when $\hat{r}$ is replaced by $\hat{r}+\gamma_{\hat{r}} \hat{r}_{\gamma}$. It is norm analytic and, by Lemma 7.7 part (i), is bounded by $c$ uniformly in the disk $\left|\gamma_{\hat{r}} \hat{r}_{\gamma}\right|_{\mathfrak{h}} \leq|\hat{r}|_{\mathfrak{h}}$. By the Cauchy formula $\left|\left(\hat{v}-\nu^{\prime} \tau-v^{\prime}\right)_{\gamma_{\hat{r}}}\right|_{\mathfrak{h}} \leq c\left|\hat{r}_{\gamma}\right|_{\mathfrak{h}}$. Since the $\gamma$ derivative of $\hat{v}-\nu^{\prime} \tau-v^{\prime}$ is bounded by the sum of these two estimates and since $\nu^{\prime}$ is independent of $\gamma$, part (i) is proved.

Proof of (ii) and (iii). Apply the same argument to $r$ as a function of $\gamma_{\hat{v}}$ and $\gamma_{\hat{r}}$. Part (iii) uses uniform bounds by $c$ on the disks $\left\|\gamma_{\hat{r}} \hat{r}_{\gamma}\right\|_{w^{2}, h} \leq c_{1}$ and $\left|\gamma_{\hat{v}} \hat{v}_{\gamma}\right|_{h} \leq c_{1}$.

Proof of Proposition 6.9. As in the proof of Theorem 6.1 all parameters are rotated by $\theta$ in the complex plane, but here we simplify notation by writing $b, \beta, \lambda, r, \Gamma$ in place of $b(\theta), \beta(\theta), \lambda(\theta), r(\theta), \Gamma(\theta)$.

Claim: Define $\mathfrak{h}_{j}$ as before (above (7.16)). There exists $c_{L}$ such that

$$
\begin{aligned}
& \left\|r_{j, \gamma}\right\|_{w, h_{j}} \leq c_{L}\left\|\Gamma\left(\beta_{j-1}\right)\right\|^{\frac{1}{2}}\left|b_{j-1}\right| \\
& \left|r_{j, \gamma}\right|_{\mathfrak{h}_{j}} \leq c_{L}\left|\lambda_{j}\right|^{2}\left\|\Gamma\left(\beta_{j-1}\right)\right\|^{3}\left|b_{j-1}\right| .
\end{aligned}
$$


By (7.11), $\hat{r}$ is a function of $\gamma_{r_{\text {main }}}$ and $\gamma_{v}$ and $\gamma_{r}$, and note that $\hat{\gamma}_{\gamma_{r_{\text {main }}}}=r_{\text {main }}, \gamma_{v}$. By Lemma 7.8, there are uniform bounds on the $w^{2}, h$ and $\mathfrak{h}$ norms of $r_{\text {main }}$ in the disk $\left|\gamma_{r_{\text {main }}}\right||b| \leq c h^{-4}$. By the Cauchy estimate and (7.11),

$$
\left\|r_{\text {main }, \gamma_{v}}\right\|_{w^{2}, h} \leq c_{L}\|C\|^{2}|b|, \quad\left|r_{\text {main }, \gamma_{v}}\right|_{\mathfrak{h}} \leq c_{L}\|C\|^{3}|\lambda|^{2}|b| .
$$

We obtain the same estimates on the $\gamma_{v}$ derivative of $\hat{r}$ because $(7.13,7.14)$ are uniform on a disk $\left|\gamma_{v}\right||b| \leq c h^{-4}$.

Consider the $\gamma_{r}$ derivative of $\hat{r}$. The derivative of the second term in (7.11) is

$$
\mathcal{S} E_{q-1}(\Delta)\left(e^{-v}\right)^{\mathcal{G}_{1} \backslash\{x\}} r_{x, \gamma_{r}},
$$

where there is no sum over $x$ and $x=0$ because $r_{x, \gamma_{r}}=0$ unless $x=0$ and then $r_{x, \gamma_{r}}=r_{\gamma_{r}}$. The absence of the sum over $x$ saves a factor of $L^{4}$ when the argument leading to (7.12) is repeated so that

$$
\left\|\mathcal{S}\left(e^{-v}\right)^{\mathcal{G}_{1} \backslash\{x\}} \mathcal{S} r_{x, \gamma_{r}}\right\|_{w^{2}, 2 h} \leq c L^{-6}\left\|r_{\gamma_{r}}\right\|_{w, h},
$$

and the same factor $L^{4}$ is also saved in estimating the $\mathfrak{h}$ norm. These terms are the dominant contribution to the $\gamma_{r}$ derivative of $\hat{r}$ : recall the $w^{2}, 2 h$ estimate on the third term in (7.11) in the proof of Proposition 7.9. It is uniform on the disk $\left\|\gamma_{r} r_{\gamma_{r}}\right\|_{w, h} \leq$ $\|r\|_{w, h}$, so that the $\gamma_{r}$ derivative of this term is bounded by $c_{L}\|r\|_{w, h}\left\|r_{\gamma_{r}}\right\|_{w, h}$. This can be absorbed into a small change in the constant $c$ in $c L^{-6}\left\|r_{\gamma_{r}}\right\|_{w, h}$ because $r$ is small by (7.16). The $\gamma_{r}$ derivative of the fourth term can also be absorbed because it is down in norm by $h^{-2 q}$ with $q=1$. In summary,

$$
\begin{aligned}
& \left\|\hat{r}_{\gamma}\right\|_{w^{2}, 2 h} \leq c_{L}\|C\|^{2}|b|+c L^{-6}\left\|r_{\gamma}\right\|_{w, h} \\
& \left|\hat{r}_{\gamma}\right|_{\mathfrak{h}^{\prime}} \leq c_{L}\|C\|^{3}|\lambda|^{2}|b|+c L^{-6}\left(\mathfrak{h}^{\prime} / \mathfrak{h}\right)^{6}\left|r_{\gamma}\right|_{\mathfrak{h}}+c_{L}\|C\|^{q} h^{-2 q}\left\|r_{\gamma}\right\|_{w, h},
\end{aligned}
$$

where the second equation is obtained in the same way using the $\mathfrak{h}$ norm and $q=5$. By this estimate and parts (ii) and (iii) of Corollary [.10,

$$
\begin{aligned}
& \left\|r_{j+1, \gamma}\right\|_{w, h_{j+1}} \leq c_{L}\left\|\Gamma\left(\beta_{j}\right)\right\|^{\frac{1}{2}}\left|b_{j}\right|+c L^{-6}\left\|r_{j, \gamma}\right\|_{w, h_{j}} \\
& \left|r_{j+1, \gamma}\right|_{\mathfrak{h}_{j+1}} \leq c_{L}\left\|\Gamma\left(\beta_{j}\right)\right\|^{3}\left|\lambda_{j}\right|^{2}\left|b_{j}\right|+c L^{-6}\left(\mathfrak{h}_{j+1} / \mathfrak{h}_{j}\right)^{6}\left|r_{j, \gamma}\right|_{\mathfrak{h}_{j}}+c_{L}\left\|\Gamma_{j}\right\|^{q} h_{j}^{-2 q}\left\|r_{j, \gamma}\right\|_{w, h_{j}},
\end{aligned}
$$

where, in the first estimate, the term $\|\hat{r}\|_{w^{2}, h}\left|\hat{v}_{\gamma}\right|_{h}$ of Corollary 7.10 was absorbed by a change of constant into $c_{L}\|\Gamma(\beta)\|^{1 / 2}|b|$, using Claim $1^{\prime}$ and $|\lambda|\left|\hat{v}_{\gamma}\right|_{h} \leq c|b|$. Likewise, in the second estimate, the term $|\hat{r}|_{\mathfrak{h}}\left|\hat{v}_{\gamma}\right|_{\mathfrak{h}}$ was absorbed into $c_{L}\|C\|^{3}|\lambda|^{2}|b|$. The claim (7.17) follows by induction (c.f. the proof of (7.16)).

By Corollary 7.10 (i), Claims 1 and $1^{\prime}$, and the estimates above on $\hat{r}_{\gamma}$,

$$
\left|\hat{v}_{\gamma}-v_{\gamma}^{\prime}\right|_{\mathfrak{h}_{j}} \leq c_{L}\left\|\Gamma_{j-1}\right\|^{3}\left|\lambda_{j-1}\right|^{2}\left|b_{j-1}\right| .
$$

We change the indices $j-1$ to $j$ on the right by increasing $c_{L}$. By the definition of $\epsilon_{*, j}$ in Section 6, $\left|\hat{v}_{\gamma}-v_{\gamma}^{\prime}\right|_{\mathfrak{h}_{j}}$ dominates $\left|\epsilon_{0, j}\right|,\left|\epsilon_{1, j}\right| \mathfrak{h}_{j}^{2}$ and $\left|\epsilon_{2, j}\right| \mathfrak{h}_{j}^{4}$. Solving the inequality for the $\epsilon_{*}$ concludes the proof of Proposition 6.2. 


\section{A Convolution of Forms and Supersymmetry}

Convolution $f, g \rightarrow \int f(u-v) g(v) d v$ of functions has a natural extension to forms. Furthermore many associated facts such as the closure of Gaussian functions under convolution also have form analogues. This is because functions and forms both pull back under the map

$$
u, v \rightarrow u-v, \quad \mathbb{C}^{N} \times \mathbb{C}^{N} \rightarrow \mathbb{C}^{N}
$$

To make this extension we first must define the integral of a form over a linear manifold of dimension less than the form. Thus, suppose $V$ is a complex linear subspace of $\mathbb{C}^{m}$ and $\omega$ is an integrable form on $\mathbb{C}^{m}$. Given any complementary subspace $V^{\perp}$ we define a form $\int_{V} \omega$ on $V^{\perp}$ by requiring that

$$
\int_{V^{\perp}} \omega^{\perp} \int_{V} \omega=\int_{\mathbb{C}^{m}} \omega^{\perp} \omega
$$

holds for all $\omega^{\perp}$ on $V^{\perp}$, where, on the right-hand side, $\omega^{\perp}$ is the form on $\mathbb{C}^{m}$ obtained by pulling back the projection from $\mathbb{C}^{m} \approx V^{\perp} \oplus V$ to $V^{\perp}$. Taking $V=\mathbb{C}^{M}$ as a subspace of $\mathbb{C}^{N+M}$ we have the following form analogue of a familiar Gaussian identity:

Proposition A.1. Let $A$ be a matrix on $\mathbb{C}^{N+M}=\mathbb{C}^{N} \times \mathbb{C}^{M}$ with positive real part. Define the quadratic form $S_{A}$ as in (2.1). Then

$$
\int_{\mathbb{C}^{M}} e^{-S_{A}}=e^{-S_{A_{u}}}
$$

where $A_{u}$ is the $N \times N$ matrix obtained by inverting $\left(A^{-1}\right)_{i j}$ with indices restricted to $1 \leq i, j \leq N$.

The partial integration over complex linear subspaces has the following desirable property:

Lemma A.2. If $\omega$ is a smooth, rapidly decaying form, then $Q \int_{V} \omega=\int_{V} Q \omega$.

Proof.

$$
\int_{V^{\perp}} \omega^{\perp} \int_{V} Q \omega=\int_{\mathbb{C}^{m}} \omega^{\perp} Q \omega
$$

Since $Q$ is a derivation and $\int Q\left(\omega^{\perp} \omega\right)=0$, the right-hand side is $-\int_{\mathbb{C}^{m}}\left(Q \omega^{\perp}\right) \omega$. Since $V$ and $V^{\perp}$ are complex linear, this is the same as

$$
-\int_{V^{\perp}}\left(Q \omega^{\perp}\right) \int_{V} \omega=\int_{V^{\perp}} \omega^{\perp} Q \int_{V} \omega .
$$

Therefore,

$$
\int_{V^{\perp}} \omega^{\perp} \int_{V} Q \omega=\int_{V^{\perp}} \omega^{\perp} Q \int_{V} \omega .
$$


This proves the claim because $\omega^{\perp}$ is arbitrary.

Proof of Proposition A.1. Let $(u, v) \in \mathbb{C}^{N} \times \mathbb{C}^{M}$. $\exp \left(-S_{A}\right)$ is a Gaussian times a sum of constant forms. Since Gaussian functions remain Gaussian when variables are integrated out,

$$
\int_{\mathbb{C}^{M}} e^{-S_{A}}=e^{-u A_{u} \bar{u}} \times \text { some constant form } \omega .
$$

The covariance of a normalized Gaussian is always the inverse of the matrix in the exponent. This identifies $A_{u}$ because the covariance of the $u$ variables is not changed by integrating out $v$. By Lemma A.2, $Q\left(\exp \left(-u A_{u} \bar{u}\right) \omega\right)=0$. Multiply both sides of this equation by $\exp \left(u A_{u} \bar{u}+d u A_{u} d \bar{u} /(2 \pi i)\right)$ which is supersymmetric and therefore commutes past $Q$. Therefore,

$$
Q\left(e^{d u A_{u} d \bar{u} /(2 \pi i)} \omega\right)=0 .
$$

Since the only constant forms annihilated by $Q$ are constants,

$$
\omega=\text { const } e^{-d u A_{u} d \bar{u} /(2 \pi i)} .
$$

Therefore

$$
\int_{\mathbb{C}^{M}} e^{-S_{A}}=\text { const } e^{-u A_{u} \bar{u}-d u A_{u} d \bar{u} /(2 \pi i)} .
$$

The constant is one because the integral of both sides over $\mathbb{C}^{N}$ is one by Lemma 2.1.

Next comes the form analogue of the well known closure property of Gaussian convolutions. $S_{A}(u-v)$ denotes the pullback of $S_{A}$ by the map (A.1).

Corollary A.3.

$$
\int e^{-S_{A}(u-v)} e^{-S_{B}(v)}=e^{-S_{C}(u)},
$$

with $C^{-1}=A^{-1}+B^{-1}$. The integral is over $v \in \mathbb{C}^{N}$.

Proof. Apply Proposition A.1 noting that the left-hand side is a Gaussian form $\exp \left(-S_{\tilde{A}}\right)$ with

$$
\tilde{A}=\left[\begin{array}{cc}
A, & -A \\
-A, & A+B
\end{array}\right] \text { whose inverse is }\left[\begin{array}{cc}
A^{-1}+B^{-1}, & B^{-1} \\
B^{-1}, & B^{-1}
\end{array}\right] .
$$

We conclude with a characterization of supersymmetric forms on $\mathbb{C}$.

Lemma A.4. (i) If $\omega$ is a smooth even supersymmetric form on $\mathbb{C}$ then $\omega=f(\tau)$ for some smooth function $f$. (ii) If $\omega$ is a smooth odd degree supersymmetric form on $\mathbb{C}$ then $\omega=f(\tau)(\phi d \bar{\phi}+\bar{\phi} d \phi)$ for some smooth function $f$. 
Proof. (i) Any even form can be written as $\omega=a+b d \phi d \bar{\phi}$, where $a, b$ are functions of $\phi$. Then

$$
Q \omega=a_{\phi} d \phi+a_{\bar{\phi}} d \bar{\phi}-(2 \pi i) b \phi d \bar{\phi}-(2 \pi i) b d \phi \bar{\phi}
$$

Therefore supersymmetry implies that the partial derivatives satisfy $a_{\phi}=2 \pi i \bar{\phi} b, a_{\bar{\phi}}=$ $2 \pi i \phi b$ which implies there is $f$ such that $a=f(\phi \bar{\phi})$ and $b=(2 \pi i)^{-1} f^{\prime}(\phi \bar{\phi})$. Therefore $\omega=f(\tau)$. (ii) Away from $\phi=0$ we can write $\omega=a \phi d \bar{\phi}+b \bar{\phi} d \phi$. $Q \omega=0$ implies $a=b$ and then $Q a=0$, so $a=a(\tau)$.

\section{B Dirichlet Boundary Conditions}

$U^{\Lambda, \beta}(x)=G_{\lambda=0}^{\Lambda}(\beta, x)$ is the $\beta$ potential for the hierarchical Levy process $\omega(t)$ killed on first exit. Recall that the Hierarchical lattice is invariant under the map $L^{-1}$ which is the shift $x \longmapsto x+\mathcal{G}_{1}$, or equivalently, when $x=\left(\ldots, x_{2}, x_{1}, x_{0}\right), L^{-1} x=\left(\ldots, x_{3}, x_{3}, x_{1}\right)$. This map induces a scaling $\mathcal{S}$ that acts on Green's functions by $\mathcal{S} U(x)=L^{-2} U\left(L^{-1} x\right)$ and on $\beta$ by $L^{2} \beta=L^{2} \beta$. We also define $\Lambda / L=\{\mathcal{S} x \mid x \in \Lambda\}$.

The main result for this section is the scale decomposition

$$
U^{\Lambda}(\beta, x)=\mathcal{S} U^{\Lambda / L}\left(L^{2} \beta, x\right)+\Gamma(\beta, x)
$$

where

$$
\Gamma(\beta, x)=\frac{1}{\gamma+\beta}\left(1_{\mathcal{G}_{0}}-\frac{1}{n} 1_{\mathcal{G}_{1}}\right),
$$

and $\gamma=1$ for the four dimensional hierarchical process. This is the only property of the hierarchical Levy process that is used in the renormalization group analysis. The remainder of this section is provided for a completeness but plays no further role in the paper.

Recall from [BEI92, that conditional on jumping, the probability of jumping from $x$ to $y$ is proportional to $|x|^{-6}$. This jump law was chosen because it makes the process scale in the same way as random walk on a four dimensional simple cubic lattice, namely for $N=\infty$, we have $U^{\beta=0}(x) \propto|x|^{-2}$. The jump rate $r$ is chosen so that the constant of proportionality is one. Equation (3.4) is an immediate consequence of

\section{Proposition B.1.}

$$
U^{\Lambda, \beta}(x)=\sum_{j=0}^{N-1} \mathcal{S}^{j} \Gamma\left(\mathcal{S}^{j} \beta, x\right)+\left(r+\mathcal{S}^{N} \beta\right)^{-1} \mathcal{S}^{N} 1_{\mathcal{G}_{0}}(x)
$$

Proof. We will prove a more general result by considering a unit ball $\mathcal{G}_{1}$ with $n$ elements and

$$
q(x-y)=c|x-y|^{-\alpha},=0 \text { if } y=x
$$


Note that

$$
\int_{\mathcal{G}_{k}} q(x) d x=1-n^{k} L^{-\alpha k} .
$$

By repeating the proof of Lemma 2.2 on page 89 of [BEI92], taking into account the killing, we find

$$
\mathbb{E}^{\Lambda}(\langle\omega(t), \xi\rangle)=\exp (-t \psi(\xi))
$$

with

$$
\psi(\xi)=r-r \int_{\mathcal{G}_{N}} q(x)\langle x, \xi\rangle d x .
$$

Since $\int q d x=1$

$$
\psi(\xi)=r \int_{\mathcal{G}_{N}} q(x)[1-\langle x, \xi\rangle] d x+r \int_{\mathcal{G}_{\mathcal{G}_{N}}} q(x) d x .
$$

Case: $\xi \in \mathcal{H}_{N}$. Recall from BEI92 that the dual ball $\mathcal{H}_{j}$ is defined by: $\xi \in \mathcal{H}_{j}$ if $\langle x, \xi\rangle=1$ for every $x \in \mathcal{G}_{j}$. Therefore, for $\xi \in \mathcal{H}_{N}$,

$$
\psi(\xi)=r \int_{\mathcal{G} \backslash \mathcal{G}_{N}} q(x) d x=r n^{N} L^{-\alpha N} .
$$

Cases: $\quad \xi \in \mathcal{H}_{j} \backslash \mathcal{H}_{j+1}$, with $j=0,1, \ldots N-1$. By the calculation starting at the bottom of page 89 of [BEI92,

$$
\begin{aligned}
\int_{\mathcal{G}_{N}} q(x)[1-\langle x, \xi\rangle] d x & =c n^{j+1} L^{-\alpha(j+1)}+c \sum_{k=j+2}^{N} L^{-\alpha k}\left(n^{k}-n^{k-1}\right) \\
& =c n^{j+1} L^{-\alpha(j+1)}+\int_{\mathcal{G}_{N} \backslash \mathcal{G}_{j+1}} q(x) d x .
\end{aligned}
$$

Therefore

$$
\psi(\xi)=r c n^{j+1} L^{-\alpha(j+1)}+r \int_{\mathcal{G} \backslash \mathcal{G}_{j+1}} q(x) d x=\gamma n^{j} L^{-\alpha j} .
$$

where $\gamma=r(1+c) n L^{-\alpha}$ is the same as the $\gamma$ in [BEI92].

Therefore, using $1_{\mathcal{H}_{j}}-1_{\mathcal{H}_{j+1}}$ to isolate these cases:

$$
(\beta+\psi(\xi))^{-1}=\sum_{j=0}^{N-1}\left(\beta+\gamma n^{j} L^{-\alpha j}\right)^{-1}\left(1_{\mathcal{H}_{j}}-1_{\mathcal{H}_{j+1}}\right)+\left(\beta+r n^{N} L^{-\alpha N}\right)^{-1} 1_{\mathcal{H}_{N}} .
$$


Then we invert the Fourier transform using lemma 2.1 in [BEI92],

$$
U^{\Lambda, \beta}=\sum_{j=0}^{N-1}\left(\beta+\gamma n^{j} L^{-\alpha j}\right)^{-1} n^{-j}\left(1_{\mathcal{G}_{j}}-\frac{1}{n} 1_{\mathcal{G}_{j+1}}\right)+\left(\beta+r n^{N} L^{-\alpha N}\right)^{-1} n^{-N} 1_{\mathcal{G}_{N}} .
$$

The proposition now follows by choosing $\alpha, n$ so that $n=L^{\alpha-2}$ and obtain

$$
U^{\Lambda, \beta}(x)=\sum_{j=0}^{N-1} n^{-j} L^{2 j} \Gamma\left(\beta L^{2 j}, \frac{x}{L^{j}}\right)+n^{-N} L^{2 N}\left(r+\beta L^{2 N}\right)^{-1} 1_{\mathcal{G}_{0}}\left(\frac{x}{L^{N}}\right),
$$

and then setting $\alpha=6$.

Remark Choosing $\alpha, n$ so that $n=L^{\alpha-2}$ and $n=L^{d}$ gives the canonical scaling factor $n^{-j} L^{2 j}=L^{-(d-2)}$ so that the infinite volume potential $(N \rightarrow \infty)$ with $\beta=0$ is

$$
U^{0}(x)=\sum_{j=0}^{\infty} L^{-(d-2) j} \Gamma\left(0, \frac{x}{L^{j}}\right)=\rho|x|^{-(d-2)}
$$

for $x \neq 0$ and $d>2$. We choose $r$ and thereby $\gamma=r(1+c) n L^{-\alpha}$ so that $\rho=1$. This requires, as in BEI92,

$$
\gamma=\frac{1-L^{-2}}{1-L^{4-\alpha}}, \quad r=\frac{1-L^{-2}}{1-L^{4-\alpha}} \frac{1-L^{2-\alpha}}{1-L^{-\alpha}} .
$$

\section{Calculations for Proposition 5.1}

To classify the different algebraic expressions that result from carrying out the derivatives in the formula in Lemma 5.2 we introduce the Feynman diagram notation. The diagrams

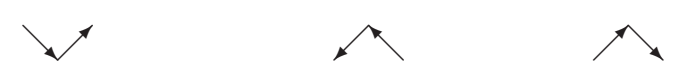

all represent $\sum \tau_{x}$. The incoming vector symbolizes the $\phi_{x}$ and the $\psi_{x}$ and the outgoing vector symbolizes the $\bar{\phi}_{x}$ and $\bar{\psi}_{x}$. The vectors are called legs. The common vertex signifies the single sum over $x$ and the sum over a term $\phi_{x} \bar{\phi}_{x}$ and a term $\psi_{x} \bar{\psi}_{x}$, whereas two vertices close together as in the two diagrams

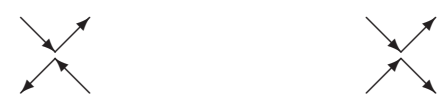

represent $\sum \tau_{x}^{2}$, in which there is a single sum over $x$ but each $\tau_{x}$ is a sum of two contributions $\phi_{x} \bar{\phi}_{x}$ and $\psi_{x} \bar{\psi}_{x}$. The action of the Laplacian $\Delta_{\Gamma}$ on $\sum \tau_{x}^{2}$ is symbolized by joining an outgoing leg to an incoming leg as in
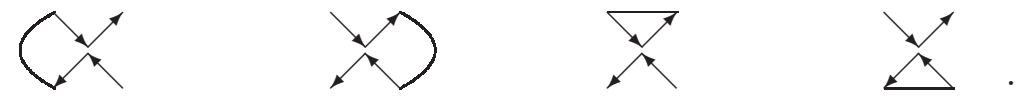
The second two diagrams each contain a closed loop. This closed loop is a factor of $\Delta_{\Gamma} \tau=0$ in the algebraic expression represented by the diagram: the anticommuting $\psi$ derivatives in $\Delta_{\Gamma}$ give a contribution that exactly cancels the contribution from the $\phi$ derivatives. Closed loops are always a factor which is the sum of two canceling contributions so diagrams containing closed loops will not be exhibited.

We classify all the terms that arise by applying $\Delta_{\Gamma}^{k}$ by drawing all possible diagrams with $k$ pairs of consistently oriented legs joined. A joined pair of legs is called a line. Each line is associated to a factor $\Gamma(x, y)$ coming from $\Delta_{\Gamma}$. Consistently, a line joining legs at the same vertex carries the factor $\Gamma(x-x)$ from $\Delta_{\Gamma}$.

Set $\gamma=0$ so that there is no observable and consider

$$
V=\sum_{y \in x+\mathcal{G}_{1}} v_{y} .
$$

By (C.1), $\Delta_{\Gamma} V$ involves two non-vanishing diagrams, but since both diagrams represent the same algebraic expression, $\lambda \Gamma(0) \sum \tau_{x}$, we write one diagram with the combinatoric coefficient 2 ,

$$
V_{1}: = e ^ { \Delta _ { \Gamma } } V = \oiiint + 2 \longdiv { \nearrow }
$$

The graphical representation for $\frac{1}{2} V_{1} \stackrel{\leftrightarrow}{\Delta}_{\Gamma} V_{1}$ is

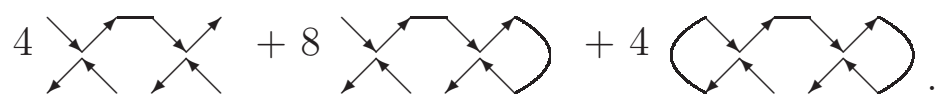

First diagram: any of 4 legs in left-hand vertex pairs with either of 2 legs in right-hand vertex and prefactor $\frac{1}{2}$. Second diagram: any of four legs pairs with one leg, prefactors $\frac{1}{2} 2$ and a factor 2 because we can interchange the 4 leg vertex with the 2 leg vertex. Third diagram: either of 2 legs can pair with one leg, prefactors $\frac{1}{2} 4$.

The graphical representation for $\frac{1}{2} \frac{1}{2 !} V_{1} \overleftrightarrow{\Delta}_{\Gamma}^{2} V_{1}$ is

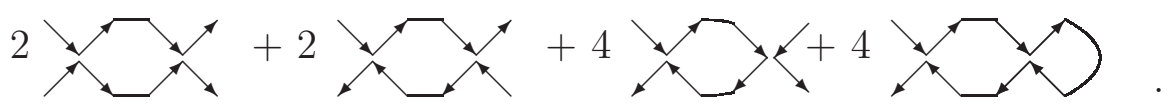

First diagram: there is already a factor of 4 in the first diagram in $\frac{1}{2} V_{1} \overleftrightarrow{\Delta}_{\Gamma} V_{1}$, there is one way to add additional line to obtain this topology, prefactor $\frac{1}{2 !}$. Second diagram: same. Third diagram: there is a factor of 4 in the second diagram in $\frac{1}{2} V_{1} \overleftrightarrow{\Delta}_{\Gamma} V_{1}$, there are two ways to add additional line to obtain this topology, prefactor $\frac{1}{2 !}$. Fourth diagram: there is a factor of 8 in the third diagram in $\frac{1}{2} V_{1} \overleftrightarrow{\Delta}_{\Gamma} V_{1}$, there is one way to add additional line to obtain this topology, prefactor $\frac{1}{2 !}$.

The graphical representation for $\frac{1}{2} \frac{1}{3 !} V_{1} \stackrel{\leftrightarrow}{\Delta}_{\Gamma}^{3} V_{1}$ is

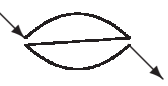


There is 2 in first diagram in $\frac{1}{2} V_{1} \overleftrightarrow{\Delta}_{\Gamma}^{2} / 2 V_{1}$, there are 2 ways to add one more line. There is no way to add an additional line to the second diagram in $\frac{1}{2} V_{1} \stackrel{\leftrightarrow}{\Delta}_{\Gamma}^{2} / 2 V_{1}$. There is 4 in third diagram in $\frac{1}{2} V_{1} \overleftrightarrow{\Delta}_{\Gamma}^{2} / 2 V_{1}$, there are 2 ways to add one more line. The total is 12 , there is prefactor $\frac{1}{3}$ in $\frac{1}{3 !}$.

For $p>3, V_{1} \overleftrightarrow{\Delta}_{\Gamma}^{p} V_{1}=0$, therefore

$$
\begin{aligned}
& \mathcal{S} \frac{1}{2} V_{1} \stackrel{\leftrightarrow}{\Delta}_{\Gamma} V_{1}=L^{-2} B_{1} \lambda^{2} \tau_{x}^{3}=0 \text { because } B_{1}=0 \\
& \mathcal{S} \frac{1}{2} \frac{1}{2 !} V_{1} \stackrel{\leftrightarrow}{\Delta}_{\Gamma}^{2} V_{1}=8 B_{2} \lambda^{2} \tau_{x}^{2}+4 B_{2} B_{0} L^{2} \lambda^{2} \tau_{x} \\
& \mathcal{S} \frac{1}{2} \frac{1}{3 !} V_{1} \stackrel{\leftrightarrow}{\Delta}_{\Gamma}^{3} V_{1}=4 B_{3} L^{2} \lambda^{2} \tau_{x}
\end{aligned}
$$

so that

$$
\begin{aligned}
& \tilde{\lambda}=\lambda-8 B_{2} \lambda^{2} \\
& \tilde{\beta}=L^{2} \beta+2 B_{0} L^{2} \lambda-4 L^{2} B_{2} B_{0} \lambda^{2}-4 L^{2} B_{3} \lambda^{2} .
\end{aligned}
$$

There are three cases to consider for the observable. Case (1) there have been fewer than $N(x)-1$ iterations so that the observable is $-\gamma b_{1, j} \phi_{0} \bar{\phi}_{x_{j}}$ with $\left|x_{j}\right|>L$. Here we know by Lemma 3.5 that $b_{i, j}=L^{-2 j}$ and $b_{1, j}=b_{2, j}=0$ and there is no need to calculate anything. Case $(2) j \geq N(x)$. For this case $v_{0}$ contains the additional terms

$$
-\gamma\left(b_{0}+b_{1} \phi_{0} \bar{\phi}_{0}+b_{2} \tau_{0} \phi_{0} \bar{\phi}_{0}+b_{3} \tau_{0}\right) \text {. }
$$

Therefore in $\mathcal{S} V_{1}$ we have the additional terms

$$
-b_{0}-b_{1} L^{-2} \phi \bar{\phi}-b_{2} L^{-4} \tau \phi \bar{\phi}-\Gamma(0) b_{1}-O\left(\Gamma^{2}\right) b_{2}-2 \Gamma(0) b_{2} \phi \bar{\phi}
$$

where we have omitted 0 and $j$ subscripts. Terms of the form $b \tau$ have been omitted for reasons explained at the end of Section 4 . In the second order part $\mathcal{S} Q_{1}$ we have additional terms

$$
O\left(\Gamma^{3}\right) \lambda b_{1}+O\left(\Gamma^{4}\right) \lambda b_{2}+\left(O\left(\Gamma^{2}\right) \lambda b_{1}+O\left(\Gamma^{3}\right) \lambda b_{2}\right) L^{-2} \phi \bar{\phi}+O\left(\Gamma^{2}\right) \lambda b_{2} L^{-4} \phi \bar{\phi} \tau .
$$

There is no $O(\Gamma) \lambda b_{1}$ contribution to $b_{2}$ because $B_{1}=0$.

Case (3) $j=N(x)-1$. This is almost the same as case (2), but with $b_{2, j}$ vanishing.

\section{References}

[AB84] M. F. Atiyah and R. Bott. The moment map and equivariant cohomology. Topology, 23:1-28, 1984. 
[BEI92] D. C. Brydges, S. Evans, and J. Z. Imbrie. Self-avoiding walk on a hierarchical lattice in four dimensions. Annals of Probability, 20:82-124, 1992.

[BI] D. C. Brydges and J. Z. Imbrie. End-to-end distance from the Green's function for a hierarchical self-avoiding walk in four dimensions. Preprint, arXiv:math-ph/0205027.

[BMM91] D. C. Brydges and I. Munoz-Maya. An application of Berezin integration to large deviations. Journal of Theoretical Probability, 4:371-389, 1991.

[LJ87] Y. Le Jan. Temps local et superchamp. Séminaire de Probabilités XXI. In Lecture Notes in Mathematics, Vol 124\%. Springer Verlag, New York, Berlin, 1987.

[Lut83] J. M. Luttinger. The asymptotic evaluation of a class of path integrals. II. J. Math. Phys., 24:2070-2073, 1983.

[McK80] A.J. McKane. Reformulation of $n \rightarrow 0$ models using anticommuting scalar fields. Physics Lett. A, 76:22-24, 1980.

[PS79] G. Parisi and N. Sourlas. Random magnetic fields, supersymmetry, and negative dimensions. Phys. Rev. Lett., 43:744-745, 1979.

[PS80] G. Parisi and N. Sourlas. Self-avoiding walk and supersymmetry. J. Phys. Lett., 41:1403-1406, 1980.

[Sim79] B. Simon. Functional integration and quantum physics. Academic Press, New York, 1979.

[Wit92] E. Witten. Two-dimensional gauge theories revisited. J. Geom. Phys., 9:303368, 1992. 